\title{
Entre science juridique et savoirs anthropologiques : évolutionnisme et his- toire comparée du droit chez Émile Jobbé-Duval (I85I-193I)
}

Résumé : Cet article brosse un portrait intellectuel de l'historien du droit Émile Jobbé-
Duval. Il examine plus particulièrement comment, dans ses travaux d'histoire comparée
du droit, l'auteur utilise la littérature anthropologique évolutionniste de la charnière des
XIX et XX siècles, et ce que ce croisement intertextuel produit. Cette contribution invite
par conséquent à réévaluer l'apport français à l'histoire de l'anthropologie juridique, sou-
vent minoré. Il semble au contraire que l'œuvre des juristes français de la Belle Époque ait
été profondément innervée, par le biais de l'intertextualité, par les réflexions anthropolo-
giques agitant la fin du siècle.

Abstract: This paper sketches the intellectual portrait of Roman law professor Émile Jobbé-Duval. It tries to analyse the way the author combines legal history with the anthropological and evolutionnist literature of the end of the Igth and the beginning of the 2oth century. This article therefore reassesses the contribution of the French jurists to the making of legal anthropology. Their works have indeed been deeply influenced by their anthropological readings, which they have mobilised by using intertextuality.

Mots clés : Jobbé-Duval, Émile - Droit-Histoire - méthode comparative - anthropologie juridique - évolutionnisme - folklore - intertextualité

Keywords : Jobbé-Duval, Émile - legal bistory - comparative law - antbropology - evolutionism - folk law - intertextuality

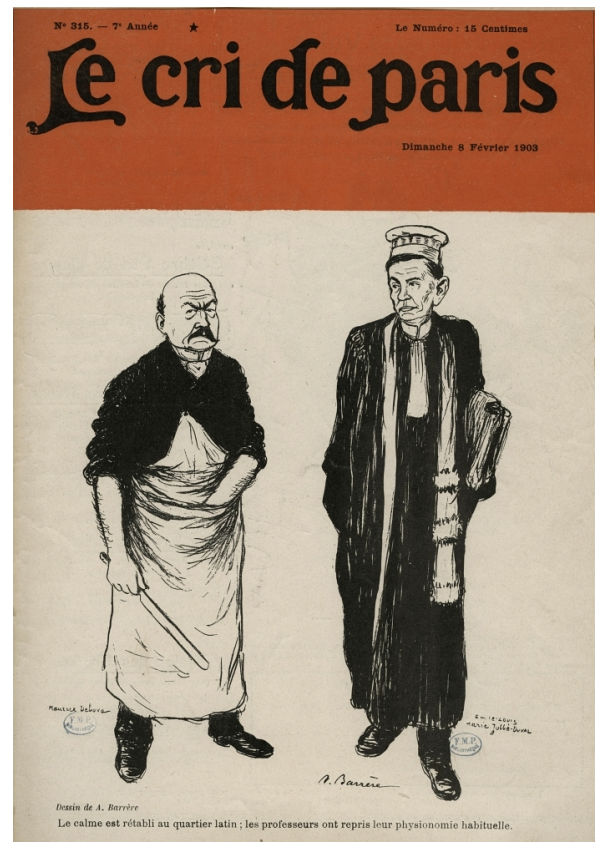

Le Cri de Paris, $n^{\circ} 315,1903$

I. En 1974, l'ethnologue Jean Poirier (192I-2009) appelait l'attention sur l'urgence du sauvetage des coutumes africaines dans un contexte de décolonisation. Pour justifier l'intérêt de ce récolement, il 
indiquait notamment que de nombreuses institutions obscures du très ancien droit romain étaient susceptibles de faire l'objet d'une interprétation renouvelée à la lumière des données africaines. Aussi concluait-il au caractère indispensable de cette collecte pour le renouvellement de la réflexion historico-juridique. Or, au cours de sa démonstration, il créditait le juriste Henri Lévy-Bruhl (I8841964) d'avoir, le premier, ouvert cette voie féconde, qui, concluait-il, commençait « tout juste à être explorée ${ }^{\gg} \gg$. Remises en perspective, ces lignes apparaissent quelque peu surprenantes... Henri LévyBruhl, premier utilisateur de la méthode comparative en histoire du droit ? Non seulement le projet scientifique du célèbre romaniste est bien plus complexe que ne veut bien l'écrire Jean Poirier ${ }^{2}$, mais l'utilisation méthodologique des droits extra-occidentaux par les historiens du droit semble remonter à la seconde moitié du XIX ${ }^{e}$ siècle. Un professeur de droit romain, plus précisément, a attaché son nom à cette tendance : Émile Jobbé-Duval (I85I-1931).

2. Né à Brest en I85I, d'un père agent de change et d'une mère fille d'un capitaine de frégate 3 , Émile Jobbé-Duval est également le neveu de l'artiste peintre républicain Félix Jobbé-Duval ${ }^{4}$. Après avoir effectué ses études secondaires au lycée de Brest, il fait son droit sur les bancs de la faculté parisienne. Inscrit au barreau de Paris, il soutient sa thèse de doctorat en $1874^{5}$, avant de décrocher le concours d'agrégation deux ans plus tard, obtenant le septième rang à 25 ans, en deçà de la moyenne nationale ${ }^{6}$. Il se marie peu après, en I877, à Marie Louise Pâris. Affecté, dans un premier temps, à Douai et à Lille, il finit par rejoindre la capitale en tant qu'agrégé en I88I, avant d'y devenir professeur adjoint en 1890 puis professeur titulaire de la chaire de droit romain deux ans plus tard 7 , un poste qu'il conserve jusqu'à sa retraite, en 1922 . Il est cependant, dès 1902, atteint d'une pneumonie qui lui vau dra des demandes répétées de congés, et dont il ne se relèvera pas. Membre fidèle de la Société de l'histoire de Paris et de l'Ile-de-France (présenté par Paul Viollet) de I886 à 1924, il est fait chevalier de la Légion d'honneur en 1902.

3. Malgré une carrière honorable et conforme aux moyennes nationales ${ }^{8}$, l'homme n'a pourtant pas imprimé une marque impérissable dans l'histoire de la discipline. Si son érudition et la qualité de ses premiers travaux lui permettent de rejoindre la faculté parisienne, il pâtit néanmoins d'une réputa-

I «Pour un programme d'urgence en ethnologie juridique », Nomos. Cabiers d'ethnologie et de sociologie juridiques, I, 1974, p. 269-280, et en particulier p. 275. Sur Jean Poirier, nous nous permettons de renvoyer à notre contribution: L. Guerlain, « Les habits neufs de l'anthropologie juridique. Relativité spatiale des concepts et fabrique disciplinaire au XX siècle », dans Penser l'ancien droit privé. Regards croisés sur les méthodes des juristes (II), dir. X. Prévost et N. Laurent-Bonne, Paris, Lextenso, 20I8, p. IOI-I3o.

2 Pour une réévaluation décisive du rapport d'Henri Lévy-Bruhl à l'ethnologie, cf. F. Audren, « Comment (ne pas) devenir un ethnologue du droit. Henri Lévy-Bruhl, les primitifs et la juristique », dans Henri Lévy-Brubl juriste et sociologue, dir. F. Audren, E. Chevreau et R. Verdier, Paris, Classiques Garnier, 2018, p. 207-247.

3 Il édite d'ailleurs les mémoires de l'aieul de son épouse : Mémoires du Bon de Bonnefoux capitaine de vaisseau, I782I85, avec une préface et des notes par Émile Jobbé-Duval, professeur à la faculté de droit de l'université de Paris, Paris, Plon, I900.

4 Les renseignements biographiques qui suivent sont tirés de ses dossiers de carrière conservés aux Archives nationales (ministère de l'Instruction Publique - F/17/22620/2 et Rectorat de Paris - AJ/16/220), de son dossier de Légion d'honneur (Archives nationales, 19800035/o187/24353) ainsi que d'un court passage que lui consacre Jacques Bouineau («Racines universitaires de Romuald Szramkiewicz (début XIX siècle-I9oo)», dans Hommage à Romuald Szramkiewicz, éd. J. Lafon, J.-L. Harouel et al., Paris, Litec, 1998, p. 392. Deux notices biographiques lui sont également consacrées: «Séance du 17 décembre $193 \mathrm{I} »$, Bulletin de la Société de législation comparée, 6I, 19311932, p. 83-84 et «Émile Jobbé-Duval », Annales de l'université de Paris, 7, 1932, p. In3. On trouvera également d'utiles renseignements dans Qui êtes-vous? Annuaire des contemporaines. Notices biographiques, Paris, Ruffy, I924, p. 404-405.

5 É. Jobbé-Duval, Étude sur la condition résolutoire en droit romain. L'bistoire du retrait lignager et la vente à réméré, Paris, Thorin, 1874.

6 Entre 1856 et I875, l'agrégation en droit est en moyenne obtenue à 27 ans et demi (V. Bernaudeau, « Origines et carrières des enseignants de la faculté de droit de Paris (XIXe-XXe siècles) », dans Paris capitale juridique (I804-1950). Étude de socio-bistoire sur la faculté de droit de Paris, dir. J.-L. Halpérin, Paris, ENS éd., 20II, p. IOo).

7 Services et titres de M. Émile Jobbé-Duval, candidat à une chaire de droit romain vacante à la faculté de droit de Paris (brochure datée de I89ı conservée à la bibliothèque Cujas à Paris).

8 Jobbé-Duval est titularisé en 1892 sur la chaire vacante de droit romain, à 4I ans, I6 ans après avoir obtenu l'agrégation, évolution de carrière tout à fait conforme aux normes de l'époque. Voir les statistiques dressées par V. Bernaudeau, « Origines et carrières des enseignants de la faculté de droit de Paris », art. cit. 
tion de piètre pédagogue9. Son entrée à la faculté de droit de Paris se déroule péniblement, JobbéDuval se faisant remarquer par l'échec cuisant de son enseignement de droit romain ${ }^{10}$. Si sa nomination en qualité de professeur-adjoint en I89o (en remplacement de Laîné) recueille certes l'unanimité des voix du conseil de faculté, c'est uniquement au motif de l'anciennetér. Malgré tout, lorsque se créé la Société d'histoire du droit en $1913^{12}$, Émile Jobbé-Duval en est nommé président. Ce qui pourrait s'interpréter comme le faîte d'une carrière exemplaire n'est en réalité qu'un choix par défaut : une nouvelle fois, cet honneur n'échoit au professeur breton qu'à l'ancienneté, en raison du décès inopiné d'Adhémar Esmein, lequel avait, le premier, émis l'idée de la création d'une telle société lors du Congrès international d'histoire comparée de 1900 et en avait, peu avant son décès en 1913, rédigé les statuts ${ }^{13}$. La faible notoriété d'Émile Jobbé-Duval est également attestée par le fait que deux notices nécrologiques seulement lui sont consacrées à sa mort, en 1922, par le Bulletin de la Société de législation comparée et les Annales de l'université de Paris ${ }^{14}$, mais aucunement par la revue-phare de sa discipline. De sa disparition, la Revue bistorique de droit français et étranger ne souffle mot...

4. Malgré le relatif oubli dont il a fait l'objet chez les historiens du droit, le cas Émile Jobbé-Duval ne manque pourtant pas d'intérêt, du point de vue de l'histoire des relations entre droit et anthropologie. De fait, les publications de ce personnage attirent immédiatement l'attention par la visible appétence de leur auteur pour les savoirs anthropologiques émergents dans la seconde moitié du $\mathrm{XIX}^{\mathrm{e}}$ siècle, qu'il tente de croiser avec sa discipline. Si Émile Jobbé-Duval ne peut pas prétendre, à l'instar d'un Henry Sumner Maine ou d'un Henri Lévy-Bruhl, prendre rang parmi les grands noms de l'anthropologie juridique, la modestie même du personnage est intéressante. Jobbé-Duval incarne en effet à merveille l'ordinaire de ce que fut la rencontre entre science juridique et savoirs anthropologiques tout au long de la Troisième République ${ }^{\text {Is }}$. Cette dernière est, au tournant du siècle, essentiellement de nature philologique. Brosser un portrait intellectuel d'Émile Jobbé-Duval équivaut à mener une enquête relative à l'utilisation de l'argument anthropologique en histoire du droit. Ce dernier se présente en réalité comme une sous-catégorie de l'argument comparatif, qui inclurait sociétés occidentales comme non-occidentales. Il s'agit, autrement dit, d'analyser la manière dont la lecture des travaux anthropologiques de son époque informe et transforme son ouvre d'historien du droit.

5. Régulièrement convoqués par les historiens du droit, les savoirs anthropologiques servent en effet fréquemment, dans la seconde moitié du XIX siècle, de réservoir argumentaire dans le cadre d'un étendard scientifique : celui de l'histoire comparée $d u$ droit, qui peut être rapportée à la grande

9 La rubrique « renseignements confidentiels » contenue dans son dossier de carrière F/I7 est, à cet égard, éloquente. Le doyen de Douai y déplore, en 1877 puis 1879 , son « ardeur excessive » et « juvénile ». Si le recteur de Douai lui reconnaît beaucoup d'érudition et d'intelligence, il écrit également que Jobbé-Duval est « desservi par son aspect imberbe et sa voix grêle : il n'a pas une très grande autorité sur les étudiants ».

Io Le doyen Charles Beudant, en I882, estime ainsi qu'il manque à son jeune collègue certaines qualités essentielles pour réussir à Paris, et en particulier des qualités d'expression orale, sans lesquelles le savoir ne saurait suffire. Il conclut cette sévère appréciation en expliquant que « les deux tiers de ses étudiants l’ont abandonné et sont allés au cours parallèle de Gérardin » (F/17/22620/2 - renseignements confidentiels du 27 mai I882).

II Académie de Paris. Faculté de droit. Conseil de la Faculté. Séance du lundi i7 novembre I890 (AJ/16/I795). Précisons tout-de-même qu'il n'y a là rien d'exceptionnel, la faculté de droit de Paris attribuant en général les promotions à l'ancienneté.

I2 Un bref compte rendu de la création de la SHD figure dans le Bulletin de la Société de législation comparée (M. Bellom, « Note sur la nouvelle Société d'histoire du droit », 43, 1913-1914, p. 125-127). Voir également la courte note que lui consacre A. Lefèbvre-Teillard, « Note sur la Société d'histoire du droit », Revue bistorique de droit français et étranger, 79, 200I-4, p. 547.

13 Selon les explications d'Émile Jobbé-Duval lui-même (Société d'histoire du droit. Allocution prononcée à l'Assemblée générale du 29 octobre I9ı3 par M. E. Jobbé-Duval au nom du Comité d'organisation, Paris, s.e., 1914, p. 5 et I3).

I4 Voir supra, note 4.

I5 Opposer ainsi les « savoirs anthropologiques » à la « science juridique » n'entend manifester aucune supériorité de la seconde sur les premières. Il s'agit simplement d'indiquer que, sous la Troisième République, l'anthropologie n'est pas encore disciplinairement constituée. On parle alors d'anthropologie physique pour désigner l'histoire naturelle de l'Homme, mais aussi d'ethnographie, plus rarement d'ethnologie ou encore de folklore. À l'inverse, le droit est depuis longtemps institutionnalisé au sein des facultés. Nous nous appuyons ici sur les remarques stimulantes de P. Macherey, tendant à distinguer savoirs et science : « Histoire des savoirs et épistémologie », Revue d'bistoire des sciences, 6o, 2007/I, p. 217-236. 
vogue du comparatisme qui agite les sciences sociales au XIX ${ }^{e}$ siècle ${ }^{16}$. Or, nul auteur n'a défendu avec plus de conviction la nécessité, pour les historiens-juristes, du comparatisme entre les sociétés « primitives » et l'histoire juridique des sociétés occidentales. Clé d'intelligibilité aussi floue qu'attirante, l'histoire comparée du droit que défendent Émile Jobbé-Duval et certains de ses collègues leur semble à même d'apporter quelques éléments de réponse aux lancinantes interrogations des historiens du droit du tournant du siècle, fascinés par les mystères des origines du droit et du primitivisme juridique ${ }^{17}$. Pour Jobbé-Duval, l'histoire comparée du droit, pivot de toute son œuvre, permet sans nul doute de mettre au jour les lois du développement juridique de l'humanité, dans le cadre conceptuel de l'évolutionnisme unilinéaire, alors dominant.

6. Aussi le portrait intellectuel du professeur breton fournit-il d'intéressants éléments de réflexion sur la réalité de ce que fut la rencontre, au tournant des XIX ${ }^{e}$ et $\mathrm{XX}^{\mathrm{e}}$ siècles, entre droit et anthropologie, ses circonstances, sa raison d'être, ses déploiements possibles, ses limites sans doute. Pour retracer l'économie et les enjeux de cette rencontre, il s'agira, dans un premier temps, d'analyser dans quelles circonstances un professeur de droit en arrive à s'initier aux savoirs anthropologiques. Nous constaterons, dans un second temps, que la majeure partie de son ouvre d'historien du droit cherche à illustrer les origines et les étapes du développement du droit, dans le cadre de l'évolutionnisme unilinéaire, développé par le biais de la méthode de l'histoire comparée du droit. Enfin, dans l'entre-deux-guerres, Jobbé-Duval prolonge sa réflexion sur le primitivisme en se mettant en quête d'une mentalité juridique primitive par le biais des études de folklore juridique.

\section{L'éveil d'un juriste aux savoirs anthropologiques}

7. Par quel mystère, par quels chemins de traverse un juriste formé à la faculté de droit dans les années 1860-1870, en pleine période exégétique, rencontre-t-il les savoirs anthropologiques naissants? Peut-on simplement expliquer cette rencontre par l'esprit du temps, le Zeitgeist ? Une telle explication, en l'occurrence, serait un peu courte : l'éveil des juristes aux sciences sociales, dans cette période de positivisme légaliste, n'a rien de naturel. Et pourtant, Émile Jobbé-Duval manifeste un intérêt précoce pour la littérature ethnologique. Il semblerait que deux facteurs se soient combinés pour permettre cet éveil : une appétence comparatiste précoce, nourrie de deux rencontres influentes.

\section{A. Appétence comparatiste et premières thématiques de recherche}

8. Dès la soutenance de sa thèse de doctorat en $\mathrm{I} 874$ sous le titre Étude sur la condition résolutoire en droit romain. L'bistoire du retrait lignager et la vente à réméré, Émile Jobbé-Duval manifeste une attention toute particulière aux législations étrangères. Dans le contexte de l'après-I870, l'impétrant, comme nombre de ses collègues, a très à cour de maîtriser la langue allemande ${ }^{18}$. Alors avocat à la Cour d'appel, il suit assidûment des cours d'allemand, à l'École pratique des hautes études, de I874 à

i6 Le comparatisme, élevé au rang de méthode par excellence par Émile Durkheim et son école, est également largement utilisé en linguistique, ou encore en histoire à partir du début du XXe siècle. Sur le comparatisme en histoire, voir É. Julien, «Le comparatisme en histoire. Rappels historiographiques et approches méthodologiques », Hypothèses, 8, 2005-I, p. 19I-20I. Rappelons par ailleurs l'intérêt bien connu de Durkheim et de son école pour le primitivisme, bien analysé par V. Karady, « Durkheim et les débuts de l'ethnologie universitaire », Actes de la recherche en sciences sociales, 74 [Recherches sur la recherche], 1988, p. 23-32. Sur la culture juridique de Durkheim, voir le récent article novateur de J.-L. Halpérin, « Durkheim et la culture juridique de son temps », dans The Sacred and the Law: the Durkheimian Legacy, dir. W. Gephart et D. Witte, Francfort-sur-le-Main, Klostermann, 2017, p. 73-92.

17 Sur le thème du primitivisme juridique, voir S. Wilf, « The Invention of Legal Primitivism », Theoretical Inquiries in Law, 10, 2009, p. 451-476 et K. Tuori, Lawyers and Savages. Ancient History and Legal Realism in the Making of Legal Antbropology, Londres, Routledge, 2015.

I8 Sur les relations entre les juristes français et allemands au XIXe siècle, cf. O. Motte, Lettres inédites de juristes français $d u X I X^{e}$ siècle conservées dans les archives et bibliothéques allemandes, Bonn, Bouvier, 1989, 2 vol. Se reporter, de manière plus générale, à C. Digeon, La crise allemande de la pensée française (I870-19I4), Paris, Presses universitaires de France, 1959. 
1876 $6^{19}$. Il y suit également les cours de critique historique dispensés par l'historien Marcel Thévenin (I843-1924), cours volontiers fréquenté par les historiens du droit, soucieux d'acquérir des compétences déjà maîtrisées par leurs collègues des facultés des lettres. Or, Thévenin était spécialiste des institutions politiques et juridiques de la France et de l'Allemagne du VI $\mathrm{I}^{\mathrm{e}}$ au IX $\mathrm{IX}^{\mathrm{e}}$ sieles ${ }^{20}$. Jobbé-Duval partage donc un intérêt certain pour l'Outre-Rhin, avec celui qui fût un véritable pilier de l'EPHE.

9. Cet attrait pour la législation comparée se prolonge par son adhésion, dès I874, à la jeune Société de législation comparée, fondée en $1869^{21}$. À partir de 1878 , notre juriste breton participe régulièrement à l'entreprise de traduction des textes de lois étrangères de l'Annuaire de législation étrangère. À la suite de son directeur de thèse Paul Gide ${ }^{22}$, qui est sans doute à l'origine de son introduction dans ce cénacle, il y traduit et présente ainsi plusieurs lois prussiennes, essentiellement relatives à la propriété et à la famille ${ }^{23}$. Parallèlement, Émile Jobbé-Duval est sollicité par différentes revues pour publier un certain nombre de recensions d'ouvrages, essentiellement dans la Revue critique de législation et de jurisprudence ${ }^{24}$, dans la Revue historique de droit français et étranger ${ }^{25}$ et dans le Bulletin de la Société de législation comparée ${ }^{26}$. Or, les ouvrages recensés sont soit des ouvrages en langue allemande, soit des ouvrages français qu'Émile Jobbé-Duval commente en les mettant en parallèle avec les législations allemande ou autrichienne, dont il est visiblement un fin connaisseur. Cette attention au comparatisme juridique, dans les années I860-1870, n'est nullement une singularité de la part d'Émile Jobbé-Duval. Bien au contraire, il s'inscrit pleinement dans la mouvance comparatiste que la fondation de la Société de législation comparée, en I869, symbolise.

I9 Registres d'inscription des étudiants pour les années I874-I875 et I875-I876 (Archives de l'EPHE) et Rapport sur l'École pratique des hautes études $1874-1875$, I-I, I874, p. I2I.

20 E. Châtelain, « Nécrologie. Marcel Thévenin », dans École pratique des hautes études. Section des sciences historiques et philologiques. Annuaire 1924-1925, Melun, Imprimerie administrative, 1924, p. 85-86.

2I Sur la Société de législation comparée, cf. M. Ancel, «Cent ans de droit comparé en France », dans Livre $d u$ centenaire de la Société de législation comparée. Un siècle de droit comparé en France (I869-1969). Les apports du droit comparé au droit positif français, Paris, LGDJ, 1969, p. 3-2I; R. Drago, « La Société de législation comparée », Ibidem, p. 25-43; É. Picard, «La Société de législation comparée de I869 à 2015 », dans Le droit comparé au $X X I^{e}$ siècle. Enjeux et défis, dir. B. Fauvarque-Cosson, Paris, Société de législation comparée, 2015, p. 49-50; D. Foussard, «Les fondateurs de la Société de législation comparée », Ibidem, p. 51-67 et Y. Falélavaki, L'bistoire d'une conversion. La doctrine française du XIX siècle et le recours à la comparaison juridique, Thèse Droit, Rennes, 2016, p. 25I et sq.

22 Notice de Paul Gide sur la base de données SIPROJURIS (http://siprojuris.symogih.org/siprojuris/enseignant/ \$6323), consultée le 4 décembre 2017.

23 É. Jobbé-Duval (professeur agrégé à la faculté de droit de Douai), « Empire d'Allemagne. Notice générale sur les travaux du Parlement allemand pendant l'année i878 », $A L E, 8$, 1878, p. 74-87; Id. (professeur agrégé à la faculté de droit de Paris), « Prusse. Loi du 2I février I88I, sur le "Höferecht" dans le cercle formé par le duché de Lauebourg. Notice, traduction et notes », Ibidem, II, I88I, p. I68-I83; Id., « Prusse. Loi du I7 janvier I883, modifiant la loi hanovrienne du 30 juin 1842 sur la procédure à suivre en matière de partage des communaux et en matière de Verkoppelung. Notice », Ibid., I3, I883, p. I76-182; Id., « Prusse. Lois agricoles. Notice », Ibid., I5, I885, p. II5-I29; enfin Id., « Prusse. Loi du 8 juin 1896 concernant l'Anerbenrecht sur les immeubles grevés de rente foncière et les biens des colons. Notice », Ibid., 26, I896, p. 176-I89.

24 É. Jobbé-Duval « Compte rendu de Féraud-Giraud, Des voies publiques et privées, modifiées, détruites ou crées par suite de l'exécution des chemins de fer », Revue critique de législation et de jurisprudence, VII, I878, p. 505.

25 É. Jobbé-Duval, «Comptes rendus bibliographiques. Revue de la législation des mines », Nouvelle revue historique de droit français et étranger, 8, I884, p. 681-684; Id., « Comptes rendus critiques. Économie politique. Georges Blondel, Étude sur les populations rurales de l'Allemagne et la crise agraire, I897 », Ibidem, 21, I897, p. 659-668; Id., «Comptes rendus critiques. Histoire du droit. F. Pinchon et E. Gourvil, Domaines congéables. Commentaire et historique de la loi du 8 février 1897, I897 », Ibid., 22, I898, p. 532-537; Id., « Comptes rendus critiques. Droit romain. Édouard Lambert, Les traditions romaines sur la succession des formes du testament devant l'bistoire comparative », Ibid., 26, 1902, p. 210-218 ; enfin Id., « Comptes rendus critiques. I. Sociologie, droit oriental et droit romain. L. Lévy-Bruhl, La mentalité primitive, $1922 »$, Ibid., 2, 1923, p. 300-308.

26 É. Jobbé-Duval, « Comptes rendus d'ouvrages. Section de la langue française. Paul Gide, Étude sur la condition privée de la femme dans le droit romain, et de la condition de l'enfant naturel et de la concubine dans la législation romaine», Bulletin de la Société de législation comparée, I4, I884-1885, p. 367-371; Id., «Comptes rendus d'ouvrages. Section des langues du Nord. Pierre Verdelot, Du bien de famille en Allemagne, et de la possibilité de son institution en France, I899 », Ibidem, 30, 1900-I90I, p. III-II3. 
Io. Parallèlement, un rapide survol des thématiques abordées par Émile Jobbé-Duval dans ses recherches ou dans ses cours apporte peut-être un élément de réponse supplémentaire. L'un de ses premiers cours en tant qu'agrégé, dispensé à la faculté de droit de Douai, est un cours complémentaire d'histoire du droit français, consacré à l'organisation de la famille et au régime des biens (I88oI88I). En outre, ses premières recensions sont fréquemment consacrées à des questions de propriété immobilière ou de droit rural. Ses lectures lui donnent ainsi l'occasion de croiser la thématique des usages ruraux, par exemple. Elles le forcent à affronter des questions qui agitent, au même moment, les savoirs anthropologiques : la question du controversé communisme primitif, ou celle des formes d'organisation de la famille, par exemple. La question de la «copropriété de la famille » le préoccupe d'ailleurs dès sa thèse de doctorat, le retrait lignager et la vente à réméré contenant tous deux cette question en filigrane ${ }^{27}$.

\section{B. Archéologie d'une influence}

II. Il semblerait surtout que le goût d'Émile Jobbé-Duval pour les savoirs anthropologiques découle d'une rencontre et d'une lecture ${ }^{28}$. Une rencontre, sans doute décisive, d'abord : celle de son directeur de thèse, le romaniste Paul Gide (1832-1880 ${ }^{29}$. Alors que, dans la première moitié du XIX siècle, l'enseignement du droit romain « dans ses rapports avec le droit français », conçu comme un complément du cours de Code civil, demeure étroitement dogmatique ${ }^{30}$, un souffle d'air frais agite la fin des années $1850^{31}$. Gide, précisément agrégé en 1859 , fait partie des premiers universitaires à historiciser l'étude du droit romain, et à en faire, non plus le prétexte commode d'un apprentissage de la technique juridique, mais un véritable objet d'histoire contextualisé. Très remarqué, son enseigne ment se signale ainsi par son souci de toujours rapprocher les règles juridiques des conceptions sociales dont elles témoignent ${ }^{32}$. Or, non seulement Gide, dans ses écrits de droit romain, émet par exemple quelques propositions d'amélioration du statut de la femme mariée, mais il est l'un des premiers historiens du droit à nourrir l'étude du droit romain de perspectives comparatistes. C'est ce dernier point, en particulier, qui marque son élève. À l'occasion de la seconde édition posthume de l'Étude de la condition privée de la fermme dans le droit ancien et moderne (I ère éd. I867;


travaux de son maître. Celui-ci, le premier, convoque en effet des matériaux assez inhabituels pour les historiens du droit du premier XIX ${ }^{e}$ siècle : récits de voyage, littérature anthropologique (John Ferguson McLennan, Lewis Morgan ${ }^{33}$, Alexis Giraud-Teulon ${ }^{34}$ ) ou encore traduction des codes brahmaniques. Jobbé-Duval rappelle que Paul Gide fût le premier, en France, à signaler la parution du célèbre ouvrage Das Mutterecht (I86I), de Johann Jakob Bachofen. Il le crédite surtout d'avoir, grâce à la mobilisation de ces différents documents, replacé l'histoire du droit dans le cadre évolutionniste qui était celui des premières grandes synthèses des années I86o : le premier, Paul Gide au-

27 É. Jobbé-Duval, Étude sur la condition résolutoire en droit romain, op. cit., p. I.

28 Sur l'importance des rencontres et de la part du hasard dans la vie intellectuelle, cf. les remarques philosophiques très éclairantes de J. Schlanger, Le neuf, le différent et le déjà-là. Une exploration de l'influence, Paris, Hermann, 2014, p. 15-23.

29 P. Ducret, « $\mathrm{v}^{\circ}$ Gide, Jean-Paul-Guillaume », dans Dictionnaire historique des juristes français, XII -XX siècle,



30 F. Audren et J.-L. Halpérin, La culture juridique française entre mythes et réalités, $X I X^{e}-X X^{e}$ siècles, Paris, CNRS éd., 2013, p. 20 et $s q$.

3 I Ibidem, p. 55 et sq.

32 P. Ducret, « $\mathrm{v}^{\circ}$ Gide, Jean-Paul-Guillaume », art. cit.

33 Lewis Morgan, en effet, fait paraître ses Systems of Consanguinity and Affinity of the Human Family en i87I et son Ancient Society, or Researches in the Line of Human Progress from Savagery, through Barbarism to Civilization en I877.

34 Licencié en droit, français mais enseignant par la suite la philosophie de l'histoire, l'esthétique et la préhistoire à l'Université de Genève, Alexis Giraud-Teulon(I839-1916) est l'auteur d'ouvrages d'histoire des institutions à caractère anthropologique, tels que La Mère chez certains peuples de l'antiquité (1867), Les origines de la famille. Questions sur les antécédents des sociétés patriarcales (1874) (en réalité un résumé explicatif de Das Mutterecht de Bachofen) ou encore L'Exogamie chez les peuples primitifs (1908). 
rait affirmé l'idée que « le droit s'est développé dans toutes les races suivant la même direction ${ }^{35}$ ». Aussi, en étudiant les coutumes exotiques, peut-on légitimement espérer retrouver l'enfance des peuples européens.

I2. Une rencontre, par conséquent, mais également une lecture. Lorsqu'il commence à écrire, au début des années i870, Émile Jobbé-Duval se place immédiatement sous le patronage du célèbre juriste anglais Henry Sumner Maine (1822-1888), premier titulaire de la chaire d'Historical and comparative jurisprudence à Oxford à partir de 1869 et responsable de la codification du droit indien ${ }^{36}$. C'est, à bien des égards, la lecture qui l'a sans doute le plus influencé. Les premiers écrits de Jobbé-Duval, qui se positionne comme un continuateur des travaux du juriste britannique, commencent invariablement par une citation de Sumner Maine et un hommage à ses recherches pionnières ${ }^{37}$. De fait, lorsque Jobbé-Duval est en train de rédiger sa thèse, au début des années i87o, l'époque est à l'effervescence de la pensée anthropologique. Entre I86I et I87I paraissent en effet plusieurs monuments de l'ethnologie juridique : Das Mutterrecht de Johann Jakob Bachofen (186I), Ancient Law d'Henry Sumner Maine (186I), Primitive marriage de John Ferguson McLennan (1865) et Primitive culture d'Edward Burnett Tylor (I87I).

13. Si Jobbé-Duval apparaît comme un fin connaisseur de cette première littérature « ethno-juridique », qu'il cite régulièrement dans ses premiers ouvrages, c'est surtout Henry Sumner Maine qui retient son attention. Faut-il vraiment s'en étonner ? La parution d'Ancient Law, en particulier, avait, en I86I, fait l'effet d'un coup de tonnerre dans les milieux autorisés. Il s'agissait, pour le Britannique, de dénoncer l'inutilité des spéculations théoriques autour de l'état de nature et du contrat social, théories jusqu'alors privilégiées par beaucoup de juristes français : à la méthode dogmatique française, Sumner Maine propose de substituer l'observation historique, qu'il nomme comparative jurisprudence ${ }^{38}$. Dans un contexte de sclérose d'études de droit exégétiques, accusées d'abstraction et de dogmatisme, et de sentiment de crise de la pensée française à la suite de la défaite de i87o, JobbéDuval se montre très séduit par la nouveauté méthodologique promue par son aîné, dont il lit les ouvrages avant même qu'ils ne soient traduits en français ${ }^{39}$. Dès la seconde partie de sa thèse, consacrée à l'histoire du retrait lignager, il annonce la mort définitive des « raisonnements a priori », pour louer l'avènement, dans les sciences juridiques, de la méthode d'observation issue des « sciences naturelles ». À lire Jobbé-Duval, cette nouvelle méthode, produit combiné d'études historiques et d'étude des peuples « moins avancés », aurait déjà abouti à un résultat remarquable : la preuve de l'unité de l'évolution du droit civil chez tous les peuples. Or, l'histoire du droit de la propriété foncière lui semble un terrain idéal pour vérifier cette loi d'unité : les relations de l'homme et de la terre obéiraient à des schémas universels. Jobbé-Duval ne propose pas de revenir sur cet acquis

35 É. Jobbé-Duval, « Comptes rendus d'ouvrages. Section de la langue française. Paul Gide, Étude sur la condition privée de la femme dans le droit romain $\gg$, art. cit., p. 368.

36 Pour une brève présentation de ce personnage en français, nous renvoyons à F. Audren, « $\mathrm{v}^{\circ}$ Maine, Sir Henry James Sumner (1822-1888) », dans Dictionnaire des grandes auvres juridiques, dir. O. Cayla et J.-L. Halpérin, Paris, Dalloz, 2008, p. 385-388. Voir également R. Verdier, « Henry Sumner Maine, historien et ethnologue du droit (Biographie, bibliographie et textes) », Droit et Cultures, 19, 1990, p. I49-190. En Angleterre, il faut essentiellement retenir deux ouvrages: R. Cocks, Sir Henry Maine. A study in Victorian jurisprudence, Cambridge, Cambridge University Press, 1988 et The Victorian achievement of Sir Henry Maine : a centennial reappraisal, éd. A. Diamond, Cambridge, Cambridge University Press, I99. Voir également, plus récemment, les remarquables analyses de M. Goëtzmann, La « comparative jurisprudence » de Henry Sumner Maine. Entre école analytique et école bistorique?, Mémoire M2 Philosophie, 2014.

37 É. Jobbé-Duval, Étude sur la condition résolutoire en droit romain, op. cit., p. 49-5o et Étude bistorique sur la revendication des meubles en droit français, Paris, Larose, I88o, p. I6.

38 Voir sur ce point l'analyse de son élève, P. Vinogradoff, L'enseignement de Sir Henry Maine. Leçon d'ouverture d'un cours d'bistoire du droit, faite au Collège Corpus Christi de l'Université d'Oxford le I ${ }^{\text {er }}$ mars 19o 4, Paris, Giard et Brière, 1904 (extrait de la Revue internationale de sociologie). L'auteur relève en particulier la critique très étendue de Maine des théories de Rousseau sur l'homme primitif (p. 7) et son désaccord profond avec la « façon française de traiter les questions des lois et de politique », apanage de « théoriciens déductifs » (p. 9).

39 Sur les traductions des ouvrages de Maine et leur réception par les juristes français, nous nous permettons de renvoyer à notre étude à paraître : L. Guerlain, « Pour une histoire de la traduction de la littérature juridique au XIX ${ }^{e}$ siècle. Quelques réflexions à partir de l'ethnologie juridique », dans Justement traduire: l'enjeu de la traduction juridique, dir. M. Bassano et W. Mastor, Toulouse, Presses de l'université Toulouse I Capitole, à paraître. 
de l'historiographie, imputable à Sumner Maine, Émile de Laveleye ${ }^{40}$ et Paul Viollet, mais simplement de le prolonger, en tentant de « faire assister à la génération graduelle du droit de propriété individuelle $e^{41} \gg$. C'est dire que Jobbé-Duval adhère, lui aussi, à la thèse de l'antériorité de la propriété collective sur la propriété individuelle ${ }^{42}$.

\section{II. Évolutionnisme et histoire comparée du droit}

I4. Toute la réflexion d'Émile Jobbé-Duval se situe par conséquent dans le cadre de l'évolutionnisme unilinéaire dans lequel l'avaient plongé ses premières lectures d'étudiant. Ce «moment ${ }^{43}$ » évolutionniste, qui se singularise par une façon entièrement nouvelle, par rapport au siècle des Lumières, de penser l'évolution sociale ${ }^{44}$, imprègne profondément la pensée du romaniste. Entre politique et génétique, la croyance en une échelle continue de progrès, sur laquelle tous les peuples pourraient être replacés en fonction de leur degré d'évolution, demeure un postulat auquel JobbéDuval ne renoncera jamais. Mieux : cette évolution uniforme peut être retracée à l'aide de l'étude des phénomènes juridiques, autre façon d'affirmer l'importance du droit pour la constitution d'une anthropologie générale. À cet égard, entre pensée analogique et intertextualité, l'histoire comparée du droit se présente comme la méthode la plus à même de reconstituer les étapes du développement juridique de l'humanité.

\section{A. L'évolutionnisme unilinéaire, entre génétique et politique}

I5. « Comment des différences perçues dans le présent peuvent-elles servir à penser des différences dans le temps ? » : tel est, formulé par Alain Testart, le grand problème théorique que pose l'anthropologie sociale évolutionniste au XIX siècle. Comment, autrement dit, une dualité synchrone sert-elle à penser une diachronie, c'est-à-dire une évolution 45 ? Dans ce que l'auteur appelle le «second évolutionnisme » (1860-1914), l'ambition consiste à essayer de « retrouver quelque chose d'une époque ancienne à partir de phénomènes contemporains », cette époque passée n'étant connue par aucune donnée directe ${ }^{46}$. Par-delà les controverses, parfois vives, entre les principaux tenants de l'évolutionnisme que sont Sumner Maine, Morgan ou encore Tylor, la clef de leur raisonnement commun tient à la notion de décalage entre différents niveaux : le mythe et la religion, voire la vie sociale, n'évoluent pas, par exemple, à la même vitesse, d'après Bachofen. Dès lors, ce décalage une fois mis au jour permettrait de reconstituer un état antérieur. Le vocabulaire ou les mythes, par exemple, seraient ainsi des conservatoires des formes anciennes de la pensée. La notion de survivance, théorisée par Edward Tylor, surdétermine par conséquent la réflexion des acteurs de la « décennie prodigieuse 》 I860-I870 (selon l'expression d'Alfred Louis Kroeber).

16. Sur ces bases, ce nouveau savoir naissant, qui émerge des efforts de la linguistique pour, progres sivement, proposer une réflexion plus large sur les institutions socioculturelles comme la parenté, le

40 Ce dernier (1822-I892), historien et économiste socialiste belge, publie en effet son célèbre De la propriété et de ses formes primitives en 1874 .

4I É. Jobbé-Duval, Étude sur la condition résolutoire en droit romain, op. cit., p. 49.

42 Voir, sur cette question, A. Testart, Le communisme primitif, économie et idéologie, Paris, Maison des sciences de l'Homme, 1995 .

43 Sur la notion de « moment », entendue comme une histoire relationnelle entre des œuvres affrontant un problème commun, cf. F. Worms, La philosophie en France au XXe siècle. Moments, Paris, Gallimard, 2009, spécialement p. 916.

44 Sur l'anthropologie au XVIII siècle, cf. M. Duchet, Antbropologie et bistoire au siècle des Lumières, Paris, Albin Michel, 1995, $2^{\mathrm{e}}$ éd. Alors qu'elles étaient auparavant définies par leurs manques supposés, on reconnaît désormais que les sociétés archaïques ont une organisation sociale différente, essentiellement fondée sur la parenté.

45 A. Testart, Avant l'bistoire. L'évolution des sociétés, de Lascaux à Carnac, Paris, Gallimard, 20I2, p. 9-Io.

46 Ibidem, p. 2I-22.

Clio@Thémis - nº I5, 2019 
mythe, la religion ou le droit ${ }^{47}$, s'engage dans une quête éperdue des origines ${ }^{48}$. Au sein de l'évolutionnisme, « perspective théorique qui présuppose l'existence d'un ordre immanent à l'histoire de l'humanité [visant à] dégager des lois dans l'ordre de succession des phénomènes sociaux et culturels ${ }^{49} \gg$, la tâche du savant consiste alors à reconstruire des grands schémas d'évolution, en postulant que celle-ci s'effectue dans le sens du progrès, c'est-à-dire d'une complexification croissante des formes sociales. Il s'agit, pour les premiers « anthropologues », de replacer les différents peuples et leurs institutions au sein de séquences d'évolution aboutissant, in fine, à la société occidentaleso. L'évolutionnisme, autrement dit, s'entend d'un « projet d'analyse comparative des sociétés humaines ${ }^{\mathrm{S}} \gg$, qui cherche à interpréter les institutions du point de vue de leur origine (génétisme), mais également du point de vue de leur évolution (comparatisme et classification) s2. $^{2}$

17. Les juristes n'échappent pas à cette proposition intellectuelle aboutissant à tenter de retrouver l'enfance des peuples européens chez des peuplades dites primitives ${ }^{33}$. La méthode comparative de Sumner Maine bénéficie d'un excellent accueil chez les historiens du droit français, qui la qualifient de « génétique », de «branche archéologique du droit comparés4 », d' « archéologie juridiquess », voire, parfois, de «paléontologie juridique ${ }^{56} \gg$. Séduits par cette métaphore stratigraphique, les juristes français, entre les années I86o et I88o, sont persuadés que « les lois de l'évolution juridique commencent à se dessiner » sous leurs yeux ${ }^{57}$. En témoignent, par exemple, certains travaux de Rodolphe Dareste ${ }^{58}$ ou d'Adhémar Esmein ${ }^{59}$. Émile Jobbé-Duval, pour sa part, avait épousé cette thèse de manière très précoce par rapport à certains de ses collègues. Dès 1874, il manifeste clairement sa croyance en l'unité et la direction commune de l'évolution du droit chez tous les peuples ${ }^{60}$, ainsi que son adhésion corrélative au concept de progrès social, « loi fatale des sociétés ${ }^{61} \gg$. Ses écrits regorgent

47 M. Kilani, Antbropologie. Du local au global, Paris, Armand Colin, 2009, p. 519.

48 A. Testart, Avant l'histoire. L'évolution des sociétés, op. cit., p. 35-37.

49 A.-C. Taylor, «Évolutionnisme », dans Dictionnaire de l'ethnologie et de l'antbropologie, dir. P. Bonte et M. Izard, $3^{\mathrm{e} e ́ d ., ~ P a r i s, ~ P r e s s e s ~ u n i v e r s i t a i r e s ~ d e ~ F r a n c e, ~ 2013, ~ p . ~ 269-270 . ~}$

so R. Deliège, Une histoire de l'anthropologie. Écoles, auteurs, théories, 2 éd., Paris, Seuil, 2013, p. $2 \mathrm{I}$.

$5 \mathrm{I} \quad$ M. Kilani, Anthropologie, op. cit., p. 233.

52 Ibidem, p. 23I et sq.

53 Pour un premier aperçu du moment évolutionniste chez les historiens du droit du XIX ${ }^{\mathrm{e}}$ siècle, nous nous permettons de renvoyer à notre étude, L. Guerlain, «Culture et usages des savoirs anthropologiques chez les juristes. De quelques apports de l'étude des recensions bibliographiques (XIX ${ }^{\mathrm{e}}$-XX $\mathrm{X}^{\mathrm{e}}$ siècles) », Revue d'histoire des facultés de droit, de la culture juridique, du monde des juristes et du livre juridique, 35, 2015, p. 25I-257.

54 M. L. Roquet, « Compte rendu [...] de l'ouvrage de M. Henry Sumner Maine, L'Ancien droit », Bulletin de la Société de législation comparée, $3, \mathrm{I} 873-1874$, p. 250.

55 R. de Kerallain, «Préface du traducteur», dans H. Sumner Maine, Études sur l'ancien droit et la coutume primitive, Paris, E. Thorin, I884, p. III.

56 J. Durieu de Leyritz «Préface du traducteur», dans H. Sumner Maine, Études sur l'histoire des institutions primitives, Paris, E. Thorin, I88o, p. XVI.

57 R. de Kerallain, « Préface du traducteur », art. cit., p. XII.

58 Partisan d'un évolutionnisme modéré, celui-ci est en effet l'un des premiers juristes français à étudier diverses législations anciennes ou lointaines, comme la législation égyptienne, les droits israélite et musulman, les anciens droits slave et scandinave ou encore les droits chinois et japonais. Il publie en particulier des Études d'bistoire du droit (1889) et des Nouvelles études d'bistoire du droit (1902-1906).

59 Esmein s'intéresse également beaucoup au primitivisme juridique et publie quelques articles en ce sens. Voir, par exemple, A. Esmein, « Note pour l'histoire des institutions primitives », Revue générale du droit, 8, I884, p. 5-14; $I d$., « La propriété foncière dans les poèmes homériques », Nouvelle revue historique de droit français et étranger, I4, I890, p. 82I-845; Id., « La coutume primitive dans un conte populaire », Ibidem, 24, 1900, p. 5-25; Id., «Les coutumes primitives dans les écrits des mythologues grecs et romains », Ibid., 25, I9OI, p. I2I-I35, 26, I902, p. 5-3I et II3-I46 ou encore $I d$., «Une survivance qui disparait : the Demise of the Crown Act », Annales des sciences politiques, I8, 1903, p. 97-II6.

60 É. Jobbé-Duval, Étude sur la condition résolutoire en droit romain, op. cit., p. 49.

6I Ibidem, p. I62. L'avènement de la notion de progrès est largement tributaire de la victoire des Modernes sur les Anciens. À partir du XVIII siècle en effet s'impose une temporalité évolutive : l'excellence ne résulte plus de la répétition des grands modèles du passé, mais se tourne au contraire vers l'avenir. Ce changement de paradigme autorise l'analyse des «sauvages » en termes de témoins vivants du passé occidental. L'évolutionnisme première mouture du XVIII siècle a ainsi constitué une véritable « révolution de la pensée », en sortant les sauvages de la simple curiosité pour en faire un objet d'étude digne d'intérêt scientifique (Â. Testart, Avant l'bistoire. L'évolution 
de preuves de sa croyance à l'évolutionnisme unilinéaire et à l'existence de peuples plus civilisés que d'autres ${ }^{62}$. S'il autorise sa réflexion à se déployer à partir de cette prémisse, c'est qu'il l'estime déjà démontrée par les premières grandes synthèses évolutionnistes de la décennie prodigieuse.

I8. Sous la plume du romaniste, le progrès s'entend en réalité du passage du collectivisme à l'individualisme. Rapporté au droit, le processus civilisationnel peut se résumer dans deux formules, qui forment les conclusions de sa thèse de doctorat : le dégagement progressif de l'individu par rapport à la famille et la liberté toujours plus grande laissée au propriétaire dans la disposition de ses biens ${ }^{63}$. On aperçoit ici clairement le positionnement politique de Jobbé-Duval, conservateur libéral, persuadé, comme beaucoup d'hommes de son temps, de la supériorité de l'individualisme juridique ${ }^{64}$ et de la propriété privée. Il n'est d'ailleurs pas surprenant que sa thèse de doctorat s'ouvre sur une citation de Sumner Maine, connu pour ses opinions libérales, relative au détachement progressif de l'individu de la dépendance de sa famille, tirée de la traduction de L'Ancien Droit par l'économiste libéral Gustave de Courcelle-Seneuil (I813-I892), également connu pour ses traductions d'Adam Smith et de John Stuart Mill's. De même, la recension d'un ouvrage de Georges Blondel relatif à la crise agraire allemande est l'occasion pour lui, en I897, d'affirmer sa méfiance envers l'interventionnisme législatif ${ }^{66}$. Comme beaucoup de ses contemporains par ailleurs, Jobbé-Duval n'est pas hostile à la colonisation. Estimant que les études d'histoire comparée du droit peuvent rendre d'utiles services à la colonisation, un texte de 1900 est l'occasion pour lui d'affirmer un certain « humanisme colonial » avant l'heure, qui sera, plus tard, la norme dans l'entre-deux-guerres ${ }^{67}$. L'histoire comparative des institutions sert, de ce point de vue, essentiellement à éviter « les malentendus [qui] peuvent être terribles entre des races que sépare une évolution de plusieurs milliers d'années ${ }^{68} \gg$. Aussi milite-t-il pour la création d'un cours d'histoire comparée des institutions et du droit à l'École coloniale, afin que les administrateurs coloniaux ne s'exagèrent pas la singularité des peuples dont ils ont la charge ${ }^{69}$. Cet enseignement, conçu pour contribuer à une colonisation éclairée, ne verra jamais le jour. Un tel positionnement politique apparaît relativement modéré par rapport aux nombreux et houleux débats qui, alimentés par la lecture de la littérature consacrée au primitivisme juridique ${ }^{70}$, font rage dans les milieux intellectuels de la fin du siècle.

des sociétés, op. cit., p. 16-22).

62 Il évoque ainsi « l'état intellectuel des races qui ne sont pas parvenues à la civilisation » (É. Jobbé-Duval, Étude bistorique sur la revendication des meubles, op. cit., p. 5-6), les « races que sépare une évolution de plusieurs milliers d'années » (Id., L'bistoire comparée du droit et l'expansion coloniale de la France, Mâcon, Protat frères, $1902-$ tiré à part extrait des Annales internationales d'bistoire, p. 6) et, encore en 1923, Id., « les sociétés inférieures actuelles » « Comptes rendus critiques. I. Sociologie, droit oriental et droit romain. L. Lévy-Bruhl, La mentalité primitive, $1922 \gg$, op. cit., p. 304).

63 É. Jobbé-Duval, Étude sur la condition résolutoire en droit romain, op. cit., p. 161 et sq.

64 Il l'affirme clairement dans un texte consacré à l'intérêt de l'étude du droit romain, en considérant que le droit du Bas-Empire, et en particulier de l'empereur Justinien, apparaît comme « plus équitable et plus respectueux des droits individuels qu'aucune autre législation de l'Antiquité » (Id., L'enseignement du droit romain. Son utilité, son état actuel, Paris, Chevalier-Maresq, 1904, p. II).

65 Voir, sur ce personnage, L. Marco, « Jean-Gustave Courcelle-Seneuil, l'orthodoxe intransigeant », dans L'économie politique en France au XIX siècle, dir. M. Lutfalla et Y. Breton, Paris, Économica, I99I, p. I4I-I6I.

66 É. Jobbé-Duval, « Comptes rendus critiques. Économie politique. Georges Blondel, Étude sur les populations rurales de l'Allemagne $\gg$, art. cit., p. 667.

67 Voir, sur ce point, B. de L'Estoile, Le goût des autres. De l'Exposition coloniale aux arts premiers, Paris, Flammarion, 20I0.

68 É. Jobbé-Duval, L'bistoire comparée du droit et l'expansion coloniale, op. cit., p. 6. Relevons, à titre incident, que Jobbé-Duval se prononce résolument contre la codification des coutumes indigènes (p. 32).

69 Ibidem, p. 31-32.

70 C'est le cas exemplaire, par exemple, de Georges Sorel, auditeur assidu des cours de Jacques Flach sur les institutions primitives au Collège de France, qui y puise des matériaux à l'appui de ses opinions socialistes (P. Rolland, « Droit, sociétés primitives et socialisme. Lettres de Georges Sorel à Jacques Flach (1900-1913) », Mil neuf cent. Revue d'bistoire intellectuelle, 29, 2011/I, p. 167-184), ou encore des nombreuses récupérations dont la théorie du matriarcat de Bachofen a pu faire l'objet. Mentionnons également le cas très célèbre de la lecture marxiste d'Ancient Society (I877) de Lewis Morgan par Friedrich Engels, qui a longtemps contribué au discrédit de cette œuvre. 


\section{B. De l'importance du droit pour la constitution d'une anthropologie générale}

19. Aboutir à une classification des sociétés sur une échelle unique de progression : voilà l'objectif de l'histoire comparée du droit selon Émile Jobbé-Duval. Cet effort de classification, qu'il laisse à d'autres (lui-même nourrit des ambitions plus modestes), aboutit à une logique circulaire : le questionnement anthropologique doit permettre de nourrir son ouvre d'historien du droit romain, laquelle peut, en retour, alimenter le matériau ethnologique. C'est donc d'une double instrumentalisation qu'il s'agit. N'écrit-il pas que l'histoire du droit fournit de précieux documents « sur les conceptions primitives de l'homme ${ }^{7 \mathrm{r}} \gg$, mais qu'en sens inverse, l'étude des peuples colonisés doit aider à la compréhension de la vie antique ${ }^{72}$ ? Son étude sur la commune annamite, parue en 1896 , lui semble propre, en replaçant ce peuple sur une échelle du progrès côte à côte avec le droit romain, à « jeter quelque lumière sur l'histoire de la propriété foncière et servir à dégager le problème des éléments qui lui sont étrangers ${ }^{73} \gg$. Il est manifeste, pour lui, que l'étude du droit romain (et en particulier du très ancien droit romain) gagne à être éclairée par des comparaisons prudentes avec d'autres peuples parvenus au même degré de civilisation. Pour Jobbé-Duval, l'enjeu consiste à replacer le peuple romain, au-delà de son caractère exceptionnel d'inventeur de la science du droit, dans « le développement des institutions qu'on rencontre dans toutes les sociétés humaines à un certain moment de leur évolution ${ }^{74} \gg$.

2o. De fait, comme Henry Sumner Maine avant lui, Jobbé-Duval tente, par ses écrits, de faire entrevoir l'importance du droit et des phénomènes juridiques pour la constitution d'une anthropologie générale, et la (re)constitution d'une histoire universelle de l'humanité. C'est en particulier l'histoire de la procédure qui permet de mesurer, selon lui, « l'état intellectuel des races qui ne sont pas encore parvenues à la civilisation $75 \gg$. Il avance pour la première fois cette idée dans son Étude bistorique sur la revendication des meubles en droit français ( $188 \mathrm{O}$ ), monographie consacrée à la façon dont la propriété mobilière fût protégée par la législation du $\mathrm{V}^{\mathrm{e}}$ au XVIII siècles $^{76}$. Jobbé-Duval creuse son intuition dans un ouvrage «très attendu ${ }^{77}$ », qui connaîtra, à sa sortie, un retentissement certain. Ses Études sur l'histoire de la procédure civile chez les Romains (1896) ${ }^{78}$ connaissent un accueil très laudatif, les commentateurs insistant sur la nouveauté des études sur l'histoire de la procédure, domaine jusqu'alors peu investi par les juristes-historiens ${ }^{79}$. De fait, selon Jobbé-Duval, l'histoire de la procédure constitue l'« un des chapitres les plus importants de l'histoire générale du droit, puisque le progrès se réalisa, en imaginant de nouveaux moyens d'agir en justice ${ }^{80} \gg$. Cette réflexion sur l'importance de la procédure provient sans doute de la formation de romaniste de JobbéDuval, qui lui a permis d'en mesurer tout le poids dans la Rome antique, particulièrement à l'époque classique, caractérisée par l'œuvre du préteur, connu pour faire évoluer le droit en accordant de nouvelles actions en justice pour des cas qui ne rentraient pas strictement dans le vieux droit civil.

7 I É. Jobbé-Duval, Étude historique sur la revendication des meubles, op. cit., p. 5.

72 Id., « La commune annamite d'après de récents travaux », Nouvelle revue historique de droit français et étranger, 20, I896, p. 6I4.

73 Ibidem, p. 615.

74 A. Audibert, « Comptes rendus critiques. Droit romain. Émile Jobbé-Duval, Étude sur l'bistoire de la procédure civile chez les Romains, t. I, 1896 », Nouvelle revue historique de droit français et étranger, 21, 1897, p. 515-525.

75 É. Jobbé-Duval, « La commune annamite d'après de récents travaux », art. cit., p. 6is.

76 Voir, pour une recension descriptive du contenu de l'ouvrage, J. Boullaire, « Comptes rendus d'ouvrages. Section de la langue française. Émile Jobbé-Duval, Étude historique sur la revendication des meubles en droit français », Bulletin de la Société de législation comparée, I0, I880-I88I, p. 391-392.

77 J. Valéry, « Bibliographie. Émile Jobbé-Duval, Études sur l'bistoire de la procédure civile chez les Romains », Revue générale du droit, XX, 1896, p. 464.

78 Seul le premier tome (É. Jobbé-Duval, La procédure par le pari (agere per sponsionem), Paris, Arthur Rousseau, I896) paraît. Le second tome annoncé ne verra jamais le jour.

79 H. Lévy-Ullmann, « Comptes rendus d'ouvrages. Section de la langue française. Émile Jobbé-Duval, Études sur l'histoire de la procédure civile chez les Romains », art. cit., p. 627.

8 o É. Jobbé-Duval, Études sur l'bistoire de la procédure civile, op. cit., p. V. 
2I. De fait, l'évolution juridique de la Rome antique fait l'objet de toutes les attentions du professeur breton : faut-il vraiment s'étonner de ce statut paradigmatique accordé à une civilisation ayant véritablement inventé la science du droit ${ }^{{ }_{1}}$ ? Quel meilleur poste d'observation pour parvenir à retracer les étapes du développement juridique de l'humanité que le peuple au sein duquel le ius est né ? Jobbé-Duval s'explique longuement sur l'intérêt tout particulier du droit romain pour son dessein, dans un article paru en 1904 dans la Revue internationale de l'enseignement ${ }^{82}$. Avoir à disposition une civilisation ayant inventé la science juridique permet, comme l'indique Jobbé-Duval, de « montrer comment naît le droit et comment il se développe par suite des incessantes transformations des sociétés humaines ${ }^{{ }^{3}} \gg$. Cette prise de position n'est pas neutre : elle intervient alors que la bataille faisait rage entre deux camps : ceux qui, d'un côté, considéraient le droit romain comme trop exceptionnel pour prendre place, sur une même échelle, aux côtés d'autres peuples sans droit, à l'instar du romaniste Charles Appleton, et ceux qui, de l'autre, en faisaient au contraire un « étalon juridique », permettant de mesurer la vie normative des autres sociétés. Émile Jobbé-Duval appartient à la seconde catégorie : non seulement le droit romain lui sert de prototype pour penser l'évolution des formes juridiques des sociétés, mais il fait également office de mesure de leur évolution tout court.

22. Aussi milite-t-il, à la suite de son maître Paul Gide, pour une nécessaire historicisation du droit romain, dont il affirme qu'il faut cesser de l'enseigner comme un modèle atemporel et comme un instrument éducatif, pour le replacer, au contraire, dans le tableau de l'histoire générale du droit ${ }^{84}$. Il importe, par conséquent, d'en exposer les règles « en distinguant avec soin suivant les époques et en recherchant quelles furent les causes sociales, politiques, économiques et morales de ses transformations successives ». C'est particulièrement, pour lui, l'histoire de l'organisation judiciaire et de la procédure qui permet de « donner le spectacle de la vie juridique ${ }^{85} »$. L'étude du droit romain, écrit Jobbé-Duval, en éclairant le « développement des sociétés humaines », sert à mieux comprendre les sociétés modernes, et en particulier les sociétés colonisées ${ }^{86}$. On note ici que le professeur breton n'affirme pas que le droit romain sert à mieux comprendre le développement juridique des sociétés humaines, mais celles-ci dans leur intégralité. C'est, en creux, l'affirmation de l'importance de la part normative de la vie des sociétés qu'il met en exergue. Le droit et son évolution disent quelque chose de la vie sociale. Ils apparaissent alors comme un observatoire privilégié du développement et des stades d'évolution des sociétés, au même titre que la linguistique ou les vestiges matériels de la vie sociale.

\section{La méthode comparatiste : pensée de l'analogie et intertextualité}

23. Remonter aux origines pour mieux comparer et classer : comment un juriste-historien de la Belle Époque met-il concrètement en ouvre l'évolutionnisme, cette « première grande solution trouvée pour ordonner la pluralité des cultures ${ }^{87} \gg$ ? Pour Émile Jobbé-Duval, la réfraction des thèses évolutionnistes dans sa pratique d'historien du droit transite par la méthode de l'histoire comparée du

8I A. Schiavone, Ius. L'invention du droit en Occident, Paris, Belin, 2008.

82 Publié sous forme de tiré-à-part : É. Jobbé-Duval, L'enseignement du droit romain. Son utilité, son état actuel, Paris, Chevalier-Maresq, 1904. Ce texte fait l'objet d'une recension descriptive: H. Lévy-Alvarès, « Comptes rendus d'ouvrages. Section de la langue française. Émile Jobbé-Duval, L'enseignement du droit romain. Son utilité, son état actuel $\gg$, Bulletin de la Société de législation comparée, 33, 1903-1904, p. 339.

83 É. Jobbé-Duval, Ibidem, p. 8.

84 De ce point de vue, il n'est sans doute pas anodin que Jobbé-Duval ait signé une étude sur le jurisconsulte humaniste François Le Douaren, les humanistes ayant été, comme on le sait, les premiers à appliquer les principes de la critique historique à l'étude du droit romain : É. Jobbé-Duval, «François Le Douaren (Duarenus), I509-1559 », dans Mélanges P. F. Girard. Études de droit romain dédiées à M. P. F. Girard à l'occasion du $60^{e}$ anniversaire de sa naissance (26 octobre I9I2), Paris, Arthur Rousseau, I9I2, t. I, p. 573-62I.

85 É. Jobbé-Duval, L'enseignement du droit romain, op. cit., p. 8-9.

86 Ibidem, p. I4.

87 G. Lenclud, «Le grand partage ou la tentation ethnologique », dans Vers une ethnologie du présent, dir. G. Althabe, D. Fabre et G. Lenclud, Paris, Éditions de la Maison des sciences de l'homme, Ministère de la Culture, 1996 [édition numérique 2015], p. Iо. 
droit, laquelle suppose une double classification analogique des sociétés et des institutions. Émile Jobbé-Duval se place explicitement sous cette bannière de l'« école de l'histoire comparée du droit $^{88} \gg$. Il s'agit là d'une allusion évidente à Henry Sumner Maine et ses disciples, mais sans doute aussi à une partie des historiens du droit français. Au sein du petit monde de l'histoire du droit à la Belle Époque, le mot d'ordre de l'histoire comparée du droit et/ou des institutions sature nombre d'écrits, sans que l'on sache à quoi correspond, au juste, cette posture méthodologique relativement floue ${ }^{89}$. Tout au plus lit-on, sous la plume des juristes-historiens, la volonté de mettre en lumière « les différents états successifs du droit dans ses branches principales » et d'en révéler la « stratification $^{90} \gg$. Or, les études d'Émile Jobbé-Duval sont régulièrement analysées comme constituant la quintessence d'une telle méthode. Henry Lévy-Ullmann, âgé de tout juste 25 ans, relève que la méthode de l'histoire comparée du droit permet à son aîné de proposer des « solutions nouvelles » dans l'interprétation du droit romain, sa « hardiesse toute magistrale » aboutissant à une « ouvre de haute généralisation ${ }^{91} \gg$. Jules Valéry ${ }^{92}$ et Adrien Audibert insistent également sur l'intérêt présenté par la méthode de l'histoire comparée du droit. Le second, en particulier, n'hésite pas à affirmer que Les études sur l'bistoire de la procédure civile chez les Romains (1896) de Jobbé-Duval constituent un « modèle » d'utilisation de cette méthode qui, maniée sans une érudition suffisamment étendue, aurait pourtant pu aboutir à des « généralisations téméraires ${ }^{93}$ ».

24. Malgré ce concert de louanges dans le monde des juristes, force est de constater qu'Émile JobbéDuval ne s'explique jamais sur la façon de mesurer la gradation des peuples sur l'échelle de la civilisa tion et du progrès. Ses écrits, au plan heuristique, s'avèrent pour le moins laconiques : l'on peine à trouver, sous sa plume, des explications méthodologiques concrètes. Un seul texte aurait pu, pourtant, s'avérer instructif : il s'agit d'une conférence prononcée en 1900, lors du Congrès d'histoire comparée de Paris, dans le cadre de la $\mathrm{II}^{e}$ section dédiée à l' « histoire comparée des institutions et du droit ${ }^{4} »$. Dans ce texte, intitulé «L'histoire comparée du droit et l'expansion coloniale de la France », Jobbé-Duval, pour la première et seule fois de sa carrière, tente de s'expliquer sur la méthode comparativess. Le Congrès d'histoire comparée lui fournit une occasion unique de penser et

88 É. Jobbé-Duval, L'bistoire comparée du droit et l'expansion coloniale, op. cit., p. 25.

89 Il y aurait indubitablement une étude d'ampleur à mener sur cette importante question pour l'histoire de la discipline. S'il est exact, comme le rappelle Jean-Louis Halpérin, que l'histoire du droit au XIX siècle est essentiellement une histoire nationale du droit, il s'agirait de savoir comment cette histoire du droit français s'articule, au plan méthodologique, avec les éléments comparatifs dont les juristes-historiens sont visiblement friands (J.-L. Halpérin, « Histoire comparée du droit », dans L'bistoire du droit en France. Nouvelles tendances, nouveaux territoires, dir. J. Krynen et B. d'Alteroche, Paris, Classiques Garnier, 2014, p. I83-20I).

90 M. L. Roquet, « Compte rendu [...] de l'ouvrage de M. Henry Sumner Maine, L'Ancien droit », art. cit., p. 250.

9I H. Lévy-Ullmann, « Comptes rendus d'ouvrages. Section de la langue française. Émile Jobbé-Duval, Études sur l'histoire de la procédure civile chez les Romains, t. I, La procédure par le pari (agere per sponsionem), I896 », Bulletin de la Société de législation comparée, 25, 1895-1896, p. 628.

92 J. Valéry, «Bibliographie. Émile Jobbé-Duval, Études sur l'histoire de la procédure civile chez les Romains », art. cit., p. 465 .

93 A. Audibert, «Comptes rendus critiques. Droit romain. Émile Jobbé-Duval, Étude sur l'bistoire de la procédure civile chez les Romains $\gg$, art. cit., p. 516 .

94 Cette section est présidée par Adhémar Esmein. On note, parmi les vice-présidents, le nom de Jacques Flach, titulaire de la chaire d'histoire des législations comparées au Collège de France (voir la contribution de Frédéric Audren dans ce numéro). Parmi les vice-présidents d'honneur figurent Ernest Glasson (spécialiste, entre autres, des institutions anglaises) et Sir F. Pollock, élève de Sumner Maine. Lors de la séance d'ouverture, Esmein annonce vouloir coupler l'histoire, science reine du XIXe siècle, avec la jeune science du droit comparé (en train de transformer le droit en science d'observation) pour aboutir à la constitution d'une « société pour l'application de la méthode comparative à l'histoire des institutions et du droit » (Annales internationales d'bistoire. Congrès de Paris Igoo. $2^{e}$ section. Histoire comparée des institutions et $d u$ droit, Paris, Armand Colin, I902, p. 5-6). A la suite de cette annonce, Esmein distribue des projets de statuts de ladite société, qui ne verra le jour qu'en I9I3, sous la dénomination plus générale de Société d'histoire du droit.

95 Ce texte important fait l'objet de deux recensions relativement descriptives. Malgré tout, on note que l'une émane d'un spécialiste du Japon et l'autre de la Revue internationale de sociologie de René Worms, preuve que l'audience des travaux de Jobbé-Duval commence à dépasser le strict champ de la science juridique : G. Appert, « Comptes rendus d'ouvrages. Section de la langue française. Compte rendu d'Émile Jobbé-Duval, L'bistoire comparée du droit et l'expansion coloniale de la France (extrait des Annales internationales d'bistoire) », Bulletin de la Société de législation comparée, 3I, I9OI-I902, p. 43I et G.-L. Duprat, « Revue des livres. I. Droit. Émile Jobbé-Duval, L’histoire 
de théoriser ce que devrait être, selon lui, une « histoire comparée du droit », qui devrait tendre à « découvrir les lois du développement de l'humanité ${ }^{96} 》$. Le moyen de découvrir ces lois, pour Jobbé-Duval, consiste à opérer un classement des institutions et sociétés. Une fois les sociétés classées par grands types et réparties sur l'échelle du progrès, l'étude d'une nouvelle société s'avère aisée. Par comparaison, il est alors facile de la placer à son tour dans le groupe lui ressemblant le plus. Il s'agit, autrement dit, d'une pensée de l'analogie. Toutefois, du fondement et du critère de cette opération de classification des sociétés, Jobbé-Duval ne s'explique nullement.

25. Au lieu de tenter d'avancer des critères de classification, il préfère en effet insister longuement sur les matériaux mobilisables pour parvenir à une telle taxinomie. L'opération classificatoire ne peut s'opérer, selon lui, qu'à condition de prendre appui sur un double matériau : les civilisations anciennes à travers les textes que celles-ci ont produit et les sociétés contemporaines pouvant faire l'objet d'une observation directe, à travers la littérature produite à leur sujet ${ }^{97}$. Et Émile Jobbé-Duval de se livrer, dans la majeure partie de son texte, à une revue de littérature consistant à synthétiser les tentatives des administrateurs coloniaux ou des savants pour connaitre les coutumes indigènes dans les différentes colonies françaises. Si ce texte s'avère une occasion manquée d'expliciter sa méthode, il nous renseigne, malgré tout, sur la volonté de Jobbé-Duval de ne pas mobiliser, dans l'exercice comparatif, uniquement les sociétés indo-européennes, mais, au contraire, de s'appuyer sur un spectre spatial plus étendu. Il insiste beaucoup sur ce dernier point ${ }^{98}$ qui avait, peut-être, manqué à Sumner Maine, lequel travaillait à partir de textes classiques, mais n'entendait pas mobiliser les apports d'une ethnographie balbutiante ${ }^{99}$.

26. Aussi le rapport qu'Émile Jobbé-Duval met en place avec les savoirs anthropologiques relève-t-il de la philologie, plaçant ainsi la dimension textuelle au premier plan de l'analyse. L'intertextualité, entendue comme la « relation de coprésence entre deux ou plusieurs textes, c'est-à-dire éidétiquement et le plus souvent, par la présence effective d'un texte dans un autre ${ }^{100} \gg$, pratiquée par le romaniste, s'exerce plus précisément par le biais de cette « relation interdiscursive primitive » qu'est la citation ${ }^{\text {10I }}$. Les écrits d’Émile Jobbé-Duval sont constellés, en notes infrapaginales, de références à caractère anthropologique. En mobilisant ainsi une littérature très disparate, le professeur breton entreprend d'annexer les enseignements de l'ethnographie à ses constructions juridiques. Assurément, l'étendue de ses lectures force l'admiration. On y trouve tous les grands noms de l'anthropologie naissante française et étrangère ${ }^{102}$, ainsi que les premiers ouvrages « ethno-juridiques » ou traitant des droits exotiques ${ }^{103}$. Jobbé-Duval circule ainsi avec aisance dans un espace littéraire profus,

comparée du droit et l'expansion coloniale de la France », Revue internationale de sociologie, 10, 1902, p. 760.

96 É. Jobbé-Duval, L'bistoire comparée du droit et l'expansion, op. cit., p. 5.

97 Ibidem, p. 6.

98 Il insiste sur l'importance de l'observation directe non seulement dans ses études (et en particulier dans l'étude précitée), mais également à l'occasion de la recension d'un ouvrage de Georges Blondel relatif à la crise agraire allemande, écrit grâce à une mission sur place financée par le Musée social (É. Jobbé-Duval, « Comptes rendus critiques. Économie politique. Georges Blondel, Étude sur les populations rurales de l'Allemagne », art. cit., p. 66o et 668).

99 C'est là l'objet de sa grande controverse avec Morgan. Malgré leur quête commune des « origines », la démarche intellectuelle des deux auteurs ne s’inscrit pas dans le même champ épistémologique. Tandis que Sumner Maine inscrit sa réflexion dans le domaine des études indo-européennes ouvert par la linguistique comparée (et limite donc les peuples étudiés, ne souhaitant que remonter aux plus anciennes institutions de l'Antiquité ou de l'Inde), Morgan et ses acolytes entendent embrasser l'expérience humaine dans son intégralité. Cette perspective plus large implique de chercher les origines, non plus seulement dans les textes du passé indo-européen, mais chez les sauvages américains, voire dans la préhistoire (A. Testart, « La question de l'évolutionnisme dans l'anthropologie sociale », Revue française de sociologie, 33-2, 1992, p. 163).

Ioo G. Genette, Palimpsestes. La littérature au second degré, Paris, Seuil, 1982, p. 8.

Ior A. Compagnon, La Seconde main ou le travail de la citation, Paris, Seuil, 1979 [réed. 2016], p. 64.

IO2 Jobbé-Duval maîtrise la littérature anglo-saxonne (Lubbock, Sumner Maine, Tylor, Colebrook sur le droit hindou) mais également germanophone (Bachofen, Ewers sur la famille primitive russe, Jacob Grimm, Albert-Hermann Post), belge (Thonissen, Emile de Laveleye), russe (Maxime Kovalevsky sur l'origine et la famille et de la propriété).

I03 Fustel de Coulanges, Giraud-Teulon, Hanoteau et Letourneux sur les coutumes kabyles, Jules Michelet sur les origines du droit français, études de droit hindou de Boscheron Desportes, Laude et de Léon Sorg, études de droit annamite de Paul d'Énjoy, Miraben, P. Ory, P.-L. Philastre, études d'Adhémar Leclère sur le droit cambodgien ou encore Ludovic Beauchet sur l'ancien droit suédois. 
dont témoigne par ailleurs le registre de ses emprunts à la bibliothèque Cujas, conservé pour les an nées I898-1928, qui apporte un éclairage supplémentaire quant aux nombreuses lectures extra-juridiques, à caractère ethnologique ou sociologique, émaillant l'œuvre du romaniste parisien ${ }^{104}$.

27. Pour autant, l'intertextualité mise en œuvre par le professeur breton se caractérise, corrélativement, par une nette indifférenciation des ressources qu'il mobilise. La praxis intertextuelle s'exerce sans aucune discrimination critique : on ne trouvera, dans les écrits d'Émile Jobbé-Duval, aucune réfutation de la théorie tardienne de l'imitation ou encore des théories durkheimiennes, à l'avènement desquelles il assiste en cette fin de siècle. Cet évolutionniste convaincu lit et mobilise ensemble Tarde (pourtant grand adversaire de l'évolutionnisme), le groupe des durkheimiens (Georges Davy, Lucien Lévy-Bruhl'os ou encore Gustave Glotz, ainsi que L'Année sociologique, qu'il emprunte régulièrement) et les théoriciens évolutionnistes (Post, Bachofen, Sumner Maine ou Kovalewski, par exemple). Comment expliquer un tel éclectisme, assorti d'une indifférence surprenante aux soubassements théoriques des écrits de ces différents groupes d'auteurs ? L'explication, en réalité, est relativement décevante. Émile Jobbé-Duval refuse de prendre parti sur les grandes controverses des sciences sociales de son temps. À propos des origines de la famille, par exemple, il écrit ne pas vouloir se prononcer sur l'antériorité du matriarcat sur le patriarcat ${ }^{106}$. Les écrits précités ne servent donc pas d'armature théorique à sa propre réflexion : il se contente d'y glaner, ici ou là, un renseignement précis sur un peuple ou une institution donnée, en faisant volontairement abstraction de l'économie générale de l'œuvre ainsi butinée. Peu lui importe donc que Tarde déconstruise l'évolutionnisme en insistant au contraire sur le rôle de l'imitation, s'il peut lui emprunter quelque donnée sur une peuplade qui l'intéresse. Cet effet de décontextualisation des œuvres ainsi convoquées au gré des besoins de la cause est d'ailleurs typique de la façon de raisonner des juristes du XIX ${ }^{\mathrm{e}}$ siècle, habitués à circuler au sein d'un système intertextuel clos sur lui-même. Il faut se garder, cependant, d'analyser cette pratique de l'intertextualité comme un simple « chapardage ouvert [...] seulement décoratif ». Pour Jobbé-Duval, cette transtextualité « construit le tissu du discours et jusqu'à son sens ». En décontextualisant ainsi les œuvres mobilisées, il est alors à même de les « [détourner] de leur portée littérale pour leur donner une portée heuristique ${ }^{107} \gg$, dans le cadre de sa pratique quotidienne d'historien du droit, profondément transformée par ses lectures ethnologiques.

28. Cette façon de pratiquer l'histoire comparée du droit de manière irénique, autrement dit sans réflexion sur le choix et la pertinence des comparaisons effectuées, est-elle propre à Émile Jobbé-Duval ? L'absence d'historiographie sur la question de l' « histoire comparée du droit » au XIX ${ }^{e}$ siècle ne rend pas la réponse aisée ${ }^{108}$. Malgré tout, un simple survol du Congrès de droit comparé de Paris, tenu du $3 \mathrm{I}$ juillet au 4 août $1900^{109}$, permet de s'apercevoir que les débats autour de l'histoire comparée du droit existent bel et bien à la Belle Époque. Ce sont en particulier deux rapports, publiés par

IO4 Registre de prêt d’Émile Jobbé-Duval (Bibliothèque Cujas, cote 29I-3). On y trouve empruntés, outre de nombreuses études de droit romain en français, italien et allemand, à peu près tous les grands noms des sciences sociales de l'époque: Gabriel Tarde, Maxime Kovalewski, Adhémar Leclère, Ihering, Savigny, Gustave Le Bon, Albert Cahuzac sur le droit malgache, Bernard sur la Nouvelle-Calédonie, Jacques Flach, Paul Ory sur la commune annamite, plusieurs ouvrages d'Albert-Hermann Post (pionnier allemand de l'ethnologie juridique), Eugen Ehrlich (pionnier autrichien de la sociologie juridique), Gustave Glotz, L'Année sociologique, Ernest Renan, les études de droit celtique d'Arbois de Jubainville, une étude sur les ordalies d'Adhémar Esmein, Starcke, Jacob Grimm, Salomon Reinach, le folkloriste Paul Sébillot, Bachofen, Joseph Kohler, Lucien Lévy-Bruhl, James Frazer, Herbert Spencer, Lubbock ou encore Georges Davy sur la foi jurée.

IO5 Auquel il consacre en 1923, dans la Revue bistorique de droit français et étranger, un long compte rendu très élogieux, réaffirmant au passage tout le profit que peuvent tirer les historiens du droit des études sociologiques : É. Jobbé-Duval, «Comptes rendus critiques. I. Sociologie, droit oriental et droit romain. L. Lévy-Bruhl, La mentalité primitive, $1922 \gg$, art. cit.

Io6 É. Jobbé-Duval, Étude sur la condition résolutoire en droit romain, op. cit., p. 56.

I07 J. Schlanger, Le neuf, le différent et le déjà-là, op. cit., p. 4I.

Io8 Sylvain Soleil, dans sa tentative de typologie des raisons de la comparaison juridique au XIX siècle, ne se saisit pas de cette question, centrale autant qu'invisible, de l'histoire comparée du droit (« Pourquoi comparait-on les droits au XIX siècle ? », Clio@Themis. Revue électronique d'histoire du droit, 13 [Du comparatisme au droit comparé, regards bistoriques], 2017, p. 13-18).

Io9 Pour une réévaluation décisive de la place de ce congrès dans l'histoire du droit comparé, cf. J.-L. Halpérin, «Associations, réseaux et ambitions nationales des comparatistes de la fin du XIX siècle à la Seconde Guerre mondiale », Ibidem, p. I-2. 
le professeur à l'université de Berlin Joseph Kohler (I849-1919) et le magistrat français Gabriel Tarde (I843-1904), qui cristallisent une opposition assez forte entre un partisan et un adversaire de l'évolutionnisme juridique. Ces deux textes développent, d'une part comme de l'autre, une argumentation méthodologique poussée sur l'(in)opportunité de l'alliance entre comparatisme et histoire en matière juridique ${ }^{\mathrm{rI}}$. Par ailleurs, Jacques Flach, titulaire de la chaire d'histoire des législations comparées au Collège de France, livre à la même époque une réflexion critique poussée et très fine sur les différents courants de l'histoire comparée du droit, leurs buts et leurs méthodes, dont il tente d'expliciter les ressorts ${ }^{\text {III }}$.

29. De ces réflexions et controverses, qui le concernent pourtant au premier chef, Jobbé-Duval ne souffle mot. Tout au plus confesse-t-il à demi-mot avoir conscience des « dangers » de la méthode comparative, tout en affirmant son caractère indispensable ${ }^{\text {Ir2 }}$. Cette phrase elliptique semble indiquer que Jobbé-Duval a connaissance des critiques croissantes adressées à cette méthode aux alentours de 1900. En réalité, l'allusion de Jobbé-Duval aux apories de l'histoire comparée du droit fait référence, ainsi que le prouve la note de bas de page afférente, à la célèbre controverse entre Charles Appleton et Édouard Lambert relative à la contestation par ce dernier de l'authenticité de la Loi des XII Tables. Sans revenir sur cette brûlante question, qui agite le monde des romanistes à la fin du siècle, signalons simplement qu'Appleton avait critiqué de manière très circonstanciée l'utilisation de la méthode comparative par son collègue lyonnais. Il contestait en particulier le fait que cette dernière soit adossée au postulat « d'une évolution entièrement uniforme chez toutes les races, dans tous les temps, sous tous les climats », une méthode encline, selon lui, à négliger les « particularités de race ou de milieu ${ }^{113} \gg$. Jobbé-Duval recense lui-même longuement, en 1902, la brochure de Lambert : s'il se montre réservé sur certaines interprétations de son collègue lyonnais, il tire prétexte de ce compte rendu pour réaffirmer sa foi dans le comparatisme. À la question de savoir si l'étude du droit romain peut bénéficier des lumières de la méthode comparative, Jobbé-Duval répond nettement par l'affirmative. Même en admettant toute la singularité d'une Rome antique ayant inventé la science juridique, cette création ne permet pas pour autant de soustraire le peuple romain « aux lois ordinaires de l'humanité $\gg$. Oui : le droit romain, aussi singulier et perfectionné soit-il, doit, et peut être interprété au moyen des données de l'histoire comparée, autre façon de dire qu'il prend lui aussi place sur une échelle continue du progrès ${ }^{\mathrm{II}}$.

3o. Si Émile Jobbé-Duval balaye ainsi d'un revers de plume les critiques adressées à la méthode de l'histoire comparée du droit, il passe également sous silence les reproches adressés à son corollaire qu'est l'évolutionnisme. Celui-ci, à partir de la fin du siècle, connaît en effet une condamnation aus si brutale ${ }^{\text {II }}$ qu'avait été vif et unanime l'engouement qu'il avait suscité quarante ans auparavant ${ }^{\mathrm{II}}$. Dans le monde du droit, certains juristes, surtout catholiques, lui reprochent sa croyance à « la religion du Progrès ${ }^{117} \gg$, qui viendrait annihiler l'idée de droit naturel pour lui substituer un évolutionnisme essentiellement matérialiste, tandis que d'autres auteurs, plus nombreux, relèvent le manque de maturité et la labilité des premiers travaux à caractère ethnologique. Leurs conclusions souvent

IIo J. Kohler, « De la méthode du droit comparé », Congrès international de droit comparé tenu à Paris du 31 juillet au 4 août 19oo. Procés-verbaux des séances et documents, Paris, LGDJ, 1905, t. I, p. 227-237 et G. Tarde, « Le droit comparé et la sociologie $\gg$, Ibidem, p. 437-445.

III J. Flach, Le Lévirat et les origines de la famille, Paris, Félix Alcan, 1900.

II2 É. Jobbé-Duval, L'enseignement du droit romain, op. cit., p. Io.

II3 Ch. Appleton, Le testament romain, la méthode du droit comparé et l'authenticité des XII Tables, Paris, A. Fontemoing, 1903, p. 3I-32.

II4 É. Jobbé-Duval, « Comptes rendus critiques. Droit romain. Édouard Lambert, Les traditions romaines sur la succession des formes du testament devant l'bistoire comparative », art. cit., p. 210-2II.

IIs Sur les raisons de la condamnation de l'évolutionnisme, cf. les explications très éclairantes d'A. Testart, « La question de l'évolutionnisme dans l'anthropologie sociale », art. cit.

II6 En raison, surtout, de la mise en ordre des nombreuses découvertes linguistiques, paléontologiques ou socioculturelles permise par cette grille de lecture, d'une redoutable efficacité heuristique (M. Panoff, Etbnologie : le deuxième souffle, Paris, Payot, I977).

II7 R. de Kerallain, « Compte rendu de Charles Letourneau, L'évolution juridique dans les diverses races humaines », Revue générale du droit, XV, I89I, p. 569. 
contradictoires ne rendent-elles pas ces jeunes études délicates à mobiliser pour l'histoire du droit ${ }^{\mathrm{II}}$ ? C'est donc une double critique, à la fois politique et méthodologique, que suscite l'évolutionnisme chez les juristes. Assurément, Émile Jobbé-Duval, de par l'étendue de ses lectures, ne pouvait ignorer ce changement de paradigme à l'œuvre dans les sciences sociales. Si, dans les années i 870 , il était pleinement un homme de son temps, en 1900, la méthode qu'il avait prônée pendant toute sa carrière et à laquelle il avait adossé l'ensemble de ses écrits, est dépassée par l'avènement de nouvelles théories explicatives, et en particulier par le diffusionnisme ${ }^{\text {II }}$.

3I. Face à cet important bouleversement intellectuel, Jobbé-Duval réagit par le silence, continuant inlassablement de promouvoir l'histoire comparée du droit. Dès 1902, il est atteint d'une grave pneumonie et multiplie les demandes de congé. Jusqu'à la Première Guerre mondiale, son activité scientifique marque alors le pas : il semble se recentrer, au début des années I900, sur des études érudites de droit romain, sans utiliser la méthode comparative ${ }^{120}$. Est-ce son état de santé qui ne lui permet plus les nombreuses lectures exigées par cette méthode ? Est-il découragé par la volée de bois vert que s'attirent ensemble l'évolutionnisme unilinéaire comme théorie et l'histoire comparée du droit comme méthode ? Sans que ces questions ne puissent recevoir de réponse certaine, il demeure tout de même que son activité scientifique connaît une seconde vie notable, dans l'entre-deuxguerres. Émile Jobbé-Duval se met alors en quête d'une mentalité juridique primitive.

\section{En quête d'une mentalité juridique primitive dans l'entre-deux-guerres}

32. Dès 1916, Émile Jobbé-Duval prend connaissance des premiers travaux de Lucien Lévy-Bruhl ${ }^{121}$ sur la mentalité primitive, dont il suit par la suite assidûment la production ${ }^{122}$. Cette lecture l'influence durablement. Convaincu de l'existence d'une mentalité juridique primitive commune à tous les peuples, il scrute et illustre cette idée à travers deux terrains : le folklore juridique breton et les croyances populaires des Romains.

\section{A. Premier terrain : le folklore juridique breton}

33. Dans l'entre-deux-guerres, l'œuvre d'Émile Jobbé-Duval opère un net tournant vers les études de folklore. Apparu au XIX siècle, ce domaine d'études étudie « les restes des anciens peuples, les superstitions et histoires qui survivent, les idées qui vivent dans notre temps, mais ne sont pas de notre temps ${ }^{123} \gg$. Prenant appui sur la théorie de la survivance, conceptualisée par l'École anthropologique

II8 Sur ces critiques, cf. L. Guerlain, « Culture et usages des savoirs anthropologiques chez les juristes », art. cit., p. 257266.

II9 B. Rupp-Eisenreich, « Diffusionnisme », dans Dictionnaire de l'ethnologie, op. cit., p. 2 oI.

I20 Explication $d u n^{\circ} 173 d u$ livre I $d u$ «De Oratore » de Cicéron, Paris, Sirey, 1905 (brochure reprenant une série d'articles parus dans la Revue historique de droit français et étranger en 1904-1905), « Explication de la loi 16 au code "de inofficioso testamento" 3, 28 », dans Mélanges Girardin, Paris, Larose et Tenin, 1907, p. 355-397 et « Histoire de la doctrine relative à la nature de la querela inofficiosi testamenti », Nouvelle revue historique de droit français et étranger, 31, 1907, p. 755-802. Ces textes font l'objet de plusieurs recensions laudatives: L. Guérin, « Comptes rendus d'ouvrages. Section de la langue française. Émile Jobbé-Duval, Explication $d u n^{\circ} 173 d u$ livre I $d u \ll D e$ oratore » de Cicéron », Bulletin de la Société de législation comparée, 35, 1905-1906, p. 191-192; Id., « Comptes rendus d'ouvrages. Section de la langue française. Émile Jobbé-Duval, Histoire de la doctrine relative à la nature de la Querela inofficiosi testamenti ; Explication de la loi 16 au Code de inofficioso testamento », Ibidem, 37, 1907-1908, p. 263-264 et, encore du même, « Comptes rendus d'ouvrages. Section de la langue française. Émile Jobbé-Duval, La nature de la Querela inofficiosi testamenti selon les jurisconsultes byzantins », Ibidem, 38, 1908-1909, p. 203.

I2I Voir, sur ce dernier, les travaux de Frédéric Keck, et en particulier Lévy-Brubl entre philosophie et anthropologie, Paris, CNRS éd., 2008.

I22 Son registre de prêt à la bibliothèque Cujas indique qu'il emprunte Les fonctions mentales dans les sociétés inférieures (I9I0) en juin 1916 puis d'octobre 1922 à janvier I923. Il emprunte également La mentalité primitive (1922) dès sa sortie, en mai-juin 1922.

I23 Selon une définition proposée en 1884 par A. Lang, rapportée par N. Belmont, «Folklore », Dictionnaire de l'ethnologie, op. cit., p. 283. 
anglaise et en particulier par Edward Tylor ${ }^{124}$, il analyse donc les « débris de civilisations mortes enclavés dans une civilisation vivante ${ }^{125} \gg$. Lorsque Jobbé-Duval investit le domaine du folklore, au début du siècle, celui-ci est structuré depuis une trentaine d'années, par plusieurs sociétés savantes et publications périodiques. Fondée par Henri Gaidoz et Eugène Rolland, la revue Mélusine existe depuis I877. La Société des traditions populaires voit le jour en I886 sous la férule de Paul Sébillot, créateur de la Revue des traditions populaires. Henri Carnoy et Émile Blémont, créent, l'année suivante, la revue La Tradition. Dans le dernier quart du XIX ${ }^{\mathrm{e}}$ siècle, certaines de ces initiatives visent à retrouver « l'expression originelle de la culture nationale préindustrielle ${ }^{\mathrm{12} 6} »$, en dégageant la « matrice celtique (celtomanie) qui est le soubassement de la culture nationale ${ }^{127} \gg$. Voilà qui ne pouvait qu'interpeller Émile Jobbé-Duval, breton d'origine. Le registre de prêt de Jobbé-Duval à la bibliothèque Cujas indique qu'il maîtrise parfaitement la littérature folklorique de son époque, et en particulier la littérature relative à la culture populaire bretonne.

34. Or, Jobbé-Duval a l'idée de décliner les études de folklore en matière juridique. Il n'est certes pas le premier juriste à s'intéresser au folklore juridique. Avant lui, cependant, les initiatives demeurent assez limitées. Le juriste et pionnier de l'ethnologie juridique croate Baltazar Bogisic (1834-1908) avait bien attiré l'attention des rédacteurs de Mélusine, en I884-1885, sur l'importance, au sein du folklore, des usages juridiques populaires ${ }^{128}$. Cette idée s'était traduite par la création d'une rubrique 《 folklore juridique » (transformée en 1900 en rubrique « droit populaire »), au sein de laquelle étaient traités des thèmes tels que le folklore juridique des enfants ${ }^{129}$, la symbolique juridique ou encore le folklore constitutionnel, essentiellement sous la plume d'Henri Gaidoz ${ }^{130}$. En I887, l' $A n$ nuaire des traditions populaires publie, sous la plume de Paul Sébillot, des «Instructions et questionnaires », dont la sixième partie s'intitule « droit folklorique ${ }^{\mathrm{i3} 1} \gg$. Ce ne sont là, cependant, que des efforts dispersés. Il faudra attendre les années 1930 pour que les études de folklore juridique prennent leur envol, sous l'impulsion du professeur de droit René Maunier, président de la Société de folklore français à partir de $1932^{132}$. Les études de folklore juridique, dans l'entre-deux-guerres, bénéficient en réalité du développement intense des études de folklore général, systématiquement réalisées, à cette période, à partir d'enquêtes de terrain ${ }^{133}$.

35. Dans ce contexte, Jobbé-Duval fait figure de maillon intermédiaire entre le folklore juridique embryonnaire des années I88o, essentiellement publié sous forme de courts entrefilets dans des revues spécialisées au gré des observations décousues des correspondants de ces périodiques, et le folklore

I24 Voir, sur ce point, N. Belmont, Paroles païennes : mythe et folklore : des frères Grimm à P. Saintyves, Paris, Imago, I986, chap. V (《L'École anthropologique anglaise et la théorie des survivances »).

I25 N. Belmont, « Folklore », dans Dictionnaire de l'ethnologie, op. cit., p. 283.

I26 G. Laferté, «L’appropriation différenciée des études folkloriques par les sociétés savantes : la science républicaine rétive au folklore? », Revue d'bistoire des sciences humaines, 20, 2009, p. 130.

I27 A. Julliard, «Folklore », dans Dictionnaire de l'historien, dir. C. Gauvard et J.-F. Sirinelli, Paris, Presses universitaires de France, 2015, p. 293.

I28 V. Bogisic, « De l'importance des usages juridiques populaires. Lettre aux Directeurs de Mélusine », Mélusine. Recueil de mythologies, littérature populaire, traditions et usages, 2, I884-1885, p. 5-8.

I29 Lequel sera ensuite repris par les travaux de Jean Carbonnier (J.-P. Baud, « Avec Jean Carbonnier, une échappée vers le folklore. En hommage à l'inventeur des jeux de droit $\gg$, dans Jean Carbonnier. L'homme et l'ouvre, dir. R. Verdier, Paris, Presses universitaires de Paris Ouest, 2012, p. 167-185).

I30 Le folkloriste André Varagnac crédite d'ailleurs Henri Gaidoz, d'avoir, le premier, mené quelques études de folklore juridique (« À propos des origines du droit », Nomos. Cabiers d'etbnologie et de sociologie juridiques, I, 1974, p. 51).

I3I P. Sébillot, «Instructions et enquêtes », Annuaire des traditions populaires, 2, I887, p. I47-I48.

I32 Membre du conseil de la Société du folklore français en I930, il en prend la présidence en 1932, pour aussitôt l'ouvrir au «folklore colonial ». Animateur majeur de la Revue de folklore français, il profite de sa position pour tenter de sensibiliser ses collègues folkloristes à un domaine relativement nouveau : le folklore juridique. C'est dans cette perspective que Maunier élabore un questionnaire, adossé au Musée des Arts et Traditions populaires (Introduction au folklore juridique. Définition, questionnaire, bibliographie, Paris, Éditions d'art et d'histoire, 1938). Destiné à servir de base de travail à des enquêtes collectives locales, ce questionnaire s'inscrit dans le double cadre de la Société de folklore français et du Comité des recherches collectives de l'Encyclopédie française, fondée en 1934 et présidée par Lucien Febvre, dont Maunier assure la vice-présidence. Sur ce questionnaire, nous nous permettons de renvoyer à notre étude : L. Guerlain, « Les habits neufs de l'anthropologie juridique », art. cit., à paraître.

133 Voir, à ce sujet, B. Müller et F. Weber, « Réseaux de correspondants et missions folkloriques. Le travail d'enquête, en France, vers $1930 »$, Gradbiva, 33, 2003, p. 43-55. 
juridique tel qu'organisé et pensé par Maunier, assis sur des enquêtes de terrain ${ }^{134}$. Son ascendance bretonne et la profusion d'études folkloriques relatives à la Bretagne expliquent qu'il choisisse cette région comme terre d'élection de son ouvrage le plus célèbre, intitulé Les idées primitives dans la Bretagne contemporaine. Essais de folklore juridique et d'histoire générale du droit, paru en deux

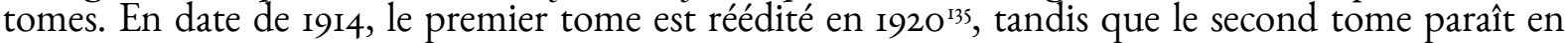
${ }_{1930^{136}}$. Le premier volet, consacré aux survivances d'ordalies dans la Bretagne des XIX et XX ${ }^{\mathrm{e}}$ siècles, était initialement paru sous forme d'articles dans la Revue bistorique de droit français et étranger, à partir de 1909. On doit à Jobbé-Duval d'avoir, en choisissant cet intitulé, rendu visible le domaine du folklore juridique. Le professeur breton est, de fait, régulièrement cité comme un pionnier de ce champ confidentiel de la recherche ${ }^{137}$.

36. Le premier tome des Idées primitives se présente comme une analyse des survivances, en terre bretonne, de ces jugements de Dieu que sont les ordalies. Jobbé-Duval propose, plus précisément, une longue relecture de l'adjuration à Saint Yves de Vérité, rituel consistant, pour une personne s'estimant lésée dans un conflit et n'ayant pas réussi à obtenir gain de cause dans un procès, à instituer Saint Yves juge de son différend, en lui enjoignant de faire mourir celui des deux adversaires dans son tort ${ }^{138}$. Le professeur breton explique disposer de suffisamment d'études de folklore pour relire cette pratique « au point de vue de l'histoire comparée du droit ${ }^{139}{ }^{\gg}$, autre façon de dire qu'il met en œuvre, ici encore, son habituelle méthode intertextuelle. Son analyse se fonde par conséquent sur les écrits de tous les folkloristes de ce début du XXe siècle, tels qu'Henri Gaidoz, Paul Sébillot, Anatole Le Braz ou encore François-Marie Luzel, pour - il reprend ici une définition d'Adhémar Esmein ${ }^{140}$ - bâtir une étude sur " "les usages extra-juridiques du peuple qui conservent tant de vieilles pratiques”, de recherches sur le folklore juridique, terme aujourd'hui consacré ${ }^{141} \gg$.

37. Loin d'essentialiser et de figer les traits de la petite patrie bretonne, Émile Jobbé-Duval explique que l'idée de cette étude lui est venue de la comparaison entre les idées sur la vie et la mort chez les Celtes et chez les Grecs anciens, grâce aux travaux de l'historien Gustave Glotz (I862-1935). Cette analogie permet à Jobbé-Duval de conclure à l'absence de caractères propres aux peuples celtiques : dès lors, en nourrissant cette première comparaison de nombreuses autres lectures, il se trouve à même de conclure à l'existence d'une universalité de la mentalité juridique primitive. Or, JobbéDuval pense, grâce à la notion de survivance de Tylor, qu'il mobilise expressément, pouvoir retrouver cet invariant en étudiant les rémanences des ordalies dans les usages bretons du XIX ${ }^{e}$ siècle, tant la Bretagne conserve encore certains aspects « des races primitives ${ }^{142} \gg$. Aussi, du point de vue de l'histoire du droit, les usages bretons offrent-ils « un tableau du très ancien droit ${ }^{143}$ ». C'est dire que la Bretagne du XIX ${ }^{e}$ siècle se présente comme un conservatoire des plus anciennes formes juridiques, propre à éclairer les juristes-historiens sur le très ancien droit. C'est à un exercice de génétique juri-

I34 Sur le rapport de Maunier à l'empirie, se reporter à l'article d'Alain Chenu, dans ce numéro.

135 É. Jobbé-Duval, L'adjuration à Saint Yves de Vérité. Les ordalies ou jugements de Dieu, Paris, Tenin, I920. La première édition est à l'heure actuelle introuvable.

I36 É. Jobbé-Duval, Études complémentaires. I. Les contrats. II. Les voux. III. La naissance. IV. Le mariage. V. Le décès, Paris, Sirey, 1930.

137 J. Carbonnier, Sociologie juridique (cours de droit, rédigé d'après la sténotypie du cours de Jean Carbonnier), s.d. (après 1961 d'après la bibliographie), p. I2 et Sociologie juridique, $3^{\mathrm{e}}$ éd., Paris, Presses universitaires de France, 2016, p. II5; H. Lévy-Bruhl, « L'ethnologie juridique. Définition et historique », dans Ethnologie générale, dir. J. Poirier, Paris, Gallimard (Encyclopédie La Pléiade), 1968, p. III9 et É. Le Roy, «Folklore juridique et Folk Law. Déplacements et enjeux d'une recherche sur « le droit des gens », Études rurales, I03-I04 [Droit et paysans], 1986, p. I4I.

I38 Pour une relecture de cette survivance, cf. T. Hamon, «L'adjuration à Saint Yves de Vérité, persistance tardive d'une ordalie populaire bretonne », Mémoires de la Société d'bistoire et d'archéologie de Bretagne, 86, 2008, p. 4188.

139 É. Jobbé-Duval, «Les idées primitives dans la Bretagne contemporaine », Nouvelle revue bistorique de droit français et étranger, 33, 1909, p. 553 .

I40 Lequel s'était quelque peu essayé au folklore général et juridique. Cf. par exemple A. Esmein, « La coutume primitive dans un conte populaire $\gg$, Ibidem, 24, 1900, p. 5-25 ou encore, du même, La Vieille Charente, chansons et croquis saintongeais, contes populaires de la Charente, Paris, Larose et Tenin, I9ro.

I4I É. Jobbé-Duval, « Les idées primitives dans la Bretagne contemporaine », art. cit., p. 553.

I42 Ibidem, p. 554 .

I43 Ibid., p. 555 .

Clio@Thémis - nI5, 2019 
dique que se livre ici le professeur parisien : remonter aux origines les plus reculées du droit, pour mieux atteindre la mentalité juridique originelle, supposée universelle. C'est, au fond, le même projet qui l'animait lorsqu'il s'aidait des coutumes des peuplades «primitives », africaines ou asiatiques, afin de les replacer sur des séquences d'évolution. Le folklore européen, dont la montée en puissance ne lui a pas échappé, lui fournit simplement un terrain supplémentaire pour parvenir à remonter aux origines du droit et en débusquer les invariants, qu'il laboure à l'aide de la méthode de l'histoire comparée du droit. La même visée anime le second tome de ses Idées primitives, consacré aux contrats, aux vœux, aux naissances, au mariage et au décès.

38. Ce croisement très extensif, dans les thèmes traités, entre histoire du droit et folklore, lui vaut un accueil remarquable de l'ouvrage. En I919, le premier tome des Idées primitives obtient en effet le prix Koenigswarter de l'Académie des sciences morales et politiques, sur une proposition de l'historien du droit Jacques Flach (I846-1919), titulaire de la chaire d'histoire des législations comparées au Collège de France, lui-même spécialiste du primitivisme juridique ${ }^{144}$. Dans son rapport, ce dernier souligne la similitude des travaux de Jobbé-Duval avec ceux du juriste Louis-Jean Koenigswarter (I814-I878), auteur d'Études historiques sur le développement des sociétés humaines (I850) tendant à démontrer l'existence d'une primitivité juridique universelle à partir de l'étude de coutumes antérieures aux lois écrites. Or, pour Flach, Koenigswarter et Jobbé-Duval aboutissent à un même résultat: celui de « rompre avec les compartiments ethniques où l'on a voulu enfermer les institutions ». Loin de valoriser un quelconque fonds commun germanique, les travaux du professeur breton tendant au contraire à aboutir à « une plus claire intelligence des institutions primitives de l'humanité $\gg$. En sus du choix d'un sujet d'études très inhabituel (en l'espèce le folklore juridique), Jacques Flach relève, parmi les mérites de l'ouvrage, la nouveauté de la méthode de Jobbé-Duval, consistant à mobiliser des lectures, ethnologiques et folkloriques, exogènes aux historiens du droit ${ }^{145}$.

39. La réédition du premier tome des Idées primitives, en 1920, est assortie d'une préface instructive quant aux visées de leur auteur. Émile Jobbé-Duval explique avoir, dans la seconde édition, ajouté le sous-titre «Essais de folklore juridique et d'histoire générale du droit », qui ne figurait pas dans la première version de l'ouvrage publiée en $1914^{146}$. L'objectif ? Faire en sorte, explique-t-il, que l'ouvrage soit visible hors de la seule sphère juridique. Force est de constater que cette stratégie fonctionne bien : les deux tomes de l'ouvrage sont abondamment commentés par les périodiques les plus divers, juridiques comme extra-juridiques. L'ouvrage fait l'objet de recensions généralement laudatives, notamment de la part de l'avocat Joseph Lefort, directeur de la Revue générale du droit très versé dans la littérature anthropologique ${ }^{147}$, qui discute longuement cet ouvrage « magistral »

I44 Cf. la contribution de Frédéric Audren dans ce numéro.

I45 J. Flach, «Rapport sur le concours pour le prix Koenigswarter à décerner en 1919 », Séances et travaux de l'Académie des sciences morales et politiques, 92, 1919, p. 250-253. On ignore au juste les liens entre Flach et JobbéDuval : tout au plus dispose-t-on d'un maigre indice consistant en une carte de visite du second adressée au premier, conservée dans le fonds Jacques Flach du Collège de France (carte de visite annotée, cote 59 CDF I3I-P). Il semblerait en outre que Jobbé-Duval nourrisse une admiration intellectuelle certaine pour Jacques Flach, comme en témoigne cette lettre de Charles Lyon-Caen, adressée à Flach, en date du 2 juin 1919 : « Voici ce que je vous avais dit au sujet de Jobbé-Duval : vous pouvez l'aviser officieusement de la proposition faite, sur votre rapport et sur votre initiative, par la section de législation. Je lui écrirai moi-même pour lui donner le même avis officieux demain ou après-demain. J'insisterai sur ce que l'initiative vient de vous. Il en sera très flatté. Car, selon ce qu'il m'a dit souvent, vous êtes un des historiens du droit qu'il tient en la plus haute et la plus légitime estime. Les récompenses tiennent beaucoup leur valeur de la valeur même de ceux qui les décernent ou les font décerner » (Collège de France, Archives Jacques Flach, $59 \mathrm{CDF}$ /97). Je remercie Frédéric Audren d'avoir attiré mon attention sur cette lettre.

I46 Il se livre d'ailleurs à une explication intéressante de ce sous-titre, en commençant par un court historique de la notion de folklore juridique, dont il attribue la paternité à Baltazar Bogisic. Il explicite également ce qu'il entend par « histoire générale du droit » : il faut, selon lui, tenir cette expression pour synonyme d'histoire comparée du droit, une méthode dont il estime que les principaux tenants, en France, sont Adhémar Esmein, Raymond Saleilles, Édouard Lambert et lui-même (É. Jobbé-Duval, Les idées primitives dans la Bretagne contemporaine, t. I, op. cit., p. I-X). Sur l'évolutionnisme d'Esmein, cf. en particulier J.-L. Halpérin, « Adhémar Esmein et les ambitions de l'histoire du droit $\gg$, Revue historique de droit français et étranger, 3, 1997, p. 428-429.

I47 Il est en effet membre du conseil d'administration de l'École d'anthropologie de Paris de Paul Broca et membre de l'Association pour l'enseignement des sciences anthropologiques. Cf, sur ce personnage, la thèse de F. Cherfouh, Le juriste entre science et politique. La Revue générale du droit, de la législation et de la jurisprudence en France et à l'étranger (I877-1938), Paris, LGDJ, 2017. 
sous forme, non de recensions, mais d'articles de fond, à la fois dans la Revue générale $d u$ droit et dans la Revue anthropologique, contribuant ainsi à la porosité entre ces deux mondes savants ${ }^{148}$. Si certains contributeurs relèvent l'intérêt de Jobbé-Duval pour les études d'histoire comparée du droit $^{149}$, plus nombreux sont ceux qui pointent le fait que Les idées primitives dépassent justement largement le strict domaine juridique, pour embrasser « la psychologie humaine $e^{150} »$, voire « l'histoire de la civilisation en général ${ }^{\mathrm{ISI}} \gg$. L'historien du droit leplaysien Frédéric Joüon des Longrais, très investi en science sociale, en recommande la lecture « au juriste, à l'historien, au sociologue, à tous ceux qu'intéresse la littérature populaire ${ }^{152} \gg$. Cette approche transdisciplinaire typique des écrits de Jobbé-Duval est également pointée par le Bulletin de la Société de législation comparée, qui qualifie l'auteur de « grand juriste », mais également d' « archéologue » et d'《 historien ${ }^{153} »$.

40. Émile Jobbé-Duval parvient ainsi à pulvériser les frontières entre le monde du droit et celui du folklore, ce dont témoigne la recension des deux tomes de l'ouvrage, en I93I, dans la Revue de folklore français, sans doute à l'instigation de René Maunier, qui venait, l'année précédente, de rejoindre le conseil de la Société du folklore français et qui prendra la tête de la revue en $1932^{154}$. Étonnamment, le même René Maunier avait livré, dix ans auparavant, une lecture très élogieuse du premier tome des Idées primitives's5. Ce fait pourrait paraître surprenant de la part de celui qui, depuis ses études ethno-juridiques sur les Kabyles jusqu'à son questionnaire de folklore juridique, ne cessait d'insister sur la nécessité de pratiquer des enquêtes de terrain, à l'opposé de la méthode philologique de Jobbé-Duval. Pour autant, il ne s'agit sans doute là que d'un moyen pour Maunier, connu pour ses recensions exigeantes, voire acerbes - notamment lors de ses débuts à la Revue internationale de sociologie de René Worms -, d'attirer l'attention sur un champ du savoir méconnu qu'il entend lui-même développer. Le seul critique, en réalité, à déplorer l'absence d'enquête de terrain et le caractère livresque de l'opus de Jobbé-Duval est Marc Bloch, lui-même versé dans la méthode comparatiste ${ }^{156}$, déçu par la banalité du folklore analysé par son collègue des facultés de droit ${ }^{157}$. Quoi qu'il en soit, l'ouvrage fait grand bruit et suscite même un émule en Roumanie, qui publie une étude suggérée par l'ouvrage de Jobbé-Duvalis8.

I48 J. Lefort, « La survivance des ordalies dans la Bretagne moderne (à propos d'É. Jobbé-Duval, Les idées primitives dans la Bretagne contemporaine. Essai de folklore juridique et d'bistoire générale du droit, 1920) », Revue générale $d u$ droit, XLV, I92I, p. I6I-I73 et « Contribution au folklore juridique. La survivance des ordalies en Bretagne », Revue anthropologique, 3I, I92I, p. 243-25I (p. I73 pour la citation).

I49 D. Buret, « Comptes rendus d'ouvrages. Section de la langue française. Compte rendu d'Émile Jobbé-Duval, Les idées primitives dans la Bretagne contemporaine. I. L'adjuration à saint Yves de Vérité, I910 », Bulletin de la Société de législation comparée, 40, I9IO-I9II, p. I70.

I5o Id., « Comptes rendus d'ouvrages. Section de la langue française. Compte rendu d'Émile Jobbé-Duval, Les idées primitives dans la Bretagne contemporaine. II. Les ordalies, I9II $\gg$, Ibidem, 4I, I9II-I9I2, p. 337.

I5I H.S., « Chronique d'histoire, de littérature et de géographie de la Bretagne. Folklore et Traditions. Compte rendu d'Émile Jobbé-Duval, Les idées primitives dans la Bretagne contemporaine (suite et fin) », Annales de Bretagne, 39, 1930-1931, p. 446. L'auteur de la recension est probablement Henri Sée.

I52 F. Joüon des Longrais, « Comptes rendus. Émile Jobbé-Duval, Les idées primitives dans la Bretagne contemporaine. Essais de folklore juridique et d'bistoire générale du droit, 1920 », Le Moyen Age. Bulletin mensuel d'histoire et de philologie, 23, 1921, p. 132-136.

I53 P. G., « Bibliographie. Section de langue française. Compte rendu d’E. Jobbé-Duval, Les idées primitives dans la Bretagne contemporaine. Essais de folklore juridique et d'bistoire générale du droit, t. II », Bulletin de la Société de législation comparée, 59-60, 1930-1931, p. 299-300.

I54 G. Brizard, « Bibliographie. Les livres. Compte rendu de Jobbé-Duval (Émile), Les idées primitives dans la Bretagne contemporaine. Essais de folklore juridique et d'bistoire générale du droit, Paris, Sirey, 1920-1930, 2 vol. in- $8^{\circ} \gg$, Revue de folklore français, 2, I931, p. 230-233.

I55 R. Maunier, « Comptes rendus critiques. Émile Jobbé-Duval, Les idées primitives dans la Bretagne contemporaine. Essais de folklore juridique et d'histoire générale du droit, 1920, seconde édition », Revue d'économie politique, 37, I923, p. 334-335. Il écrit de l'ouvrage qu'il s'agit de « l'une des meilleures contributions que la science française ait données au folklore juridique et à la sociologie comparée » (p. 335).

I56 Marc Bloch aujourd'bui. Histoire comparée ES Sciences sociales, dir. H. Atsma, A. Burguière, Paris, École des hautes études en sciences sociales, 1990.

I57 M. Bloch, «Un milieu social d'après son folklore juridique », Annales d'bistoire économique et sociale, IO, I93I, p. 286-287.

I58 H.S., «Chronique d'histoire et de littérature de Bretagne. Histoire. Compte rendu d'André Radulesco, Ressemblances entre les idées primitives dans la Bretagne contemporaine et celles du peuple roumain, Revue historique 


\section{B. Second terrain : les croyances populaires des Romains}

4I. Entre la publication des deux tomes de ses Idées primitives, Émile Jobbé-Duval fait paraître son avant-dernier ouvrage, intitulé Les morts malfaisants. Larves, lémures, d'après le droit et les croyances populaires des Romains (1924). Cet ouvrage, au thème novateur, se présente comme l'une des premières analyses de la question des morts malfaisants, revenant hanter les vivants. La thèse de Jobbé-Duval consiste à démontrer que les croyances relatives aux morts malfaisants ont exercé une influence notable sur les destinées du droit romain, et en particulier sur la question des testaments. Dès la préface de l'ouvrage, l'auteur se place immédiatement sous un double patronage : celui de James Frazer (I854-I94I) ${ }^{\text {I59 }}$ d'abord, lequel avait consacré toute son ouvre à l'analyse des mythes, croyances et rites; celui de Lucien Lévy-Bruhl (1857-1939) ensuite et de ses œuvres relatives à la mentalité primitive. On doit en effet à ces deux auteurs, explique Jobbé-Duval, d'avoir prouvé que « dans les sociétés inférieures, tout s'explique par l'intervention des puissances invisibles, et non par des lois naturelles ou par le hasard ${ }^{160} \gg$. Lier ainsi l'évolution du droit et la croyance dans le monde invisible, en 1924, apparaît indéniablement comme une nouveauté historiographique.

42. Comment Émile Jobbé-Duval en est-il venu à creuser ce sillon novateur ? Une indication nous est fournie par le début de son ouvrage, qui s'ouvre sur l'affirmation de l'existence de similitudes importantes entre les superstitions des Bas-Bretons du XIX siècle et les croyances populaires des Romain ${ }^{161}$. C'est donc en raison de sa double connaissance approfondie du folklore breton et du droit romain que Jobbé-Duval se saisit de ce sujet en vogue ${ }^{162}$. On aperçoit, une fois encore, comment sa connaissance étendue de la littérature folklorique transforme sa réflexion d'historien du droit en le menant vers des chemins inexplorés. C’est en particulier la lecture du folkloriste Henri Gaidoz qui le conduit vers l'idée qu'il n'y aurait, à travers les âges, qu'un seul folklore qui se perpétuerait inchan gé, autre manière d'affirmer que les idées primitives de l'Homme ne diffèreraient guère que sur des points de détail. Avant de développer les conséquences juridiques des superstitions romaines relatives aux âmes des défunts, Émile Jobbé-Duval propose, dans le second chapitre de son ouvrage, une théorie générale des morts malfaisants. Fidèle à sa méthode comparative, il juxtapose tous les éléments en sa possession en brassant un panel très diversifié de peuples, antiques (Hindous, Assyrie, Grèce et Égypte ancienne) ou actuels (Chine, Annam, démonologie polonaise, Cafres, Bretons, Indiens grâce à la célèbre étude du durkheimien Robert Hertz relative aux représentations collectives de la mort), afin de brosser un état des lieux de la question. De manière plus générale, ses développements relatifs au droit romain sont parsemés de comparaisons avec les croyances relatives aux morts de ces différents peuples. L'objectif de cette mobilisation étendue de la littérature relative aux âmes

de droit français et étranger, 1932, p. I-34 », Annales de Bretagne, 40, 1932-1933, p. 574.

I59 Ses lectures de Frazer sont attestées par son registre de prêt à la bibliothèque Cujas, qui indique qu'il emprunte le tome I du Rameau d'or (1898) d'octobre 1918 à janvier 1919 et Les origines magiques de la royauté (1920) en janvier 1922. Toutefois, de manière surprenante, aucun ouvrage de Frazer n'est cité dans son ouvrage, ce qui lui sera reproché par le folkloriste Pierre Saintyves, qui, huit ans plus tard, écrira lui-même un texte relatif au folklore juridique, poussé en cela par René Maunier (P. Saintyves, «Compte rendu d'Émile Jobbé-Duval, Les morts malfaisants. Larves, lémures, d'après le droit et les croyances populaires des Romains, 1924 », Revue anthropologique, 35, I925, p. 402-403). Son texte sur le folklore juridique, issu d'une conférence prononcée à la salle d'ethnologie juridique de la faculté de droit de Paris, est publié dans la collection des Études de sociologie et d'etbnologie juridiques fondée par René Maunier (P. Saintyves, « Le folklore juridique », Salle de travail d'etbnologie juridique. Faculté de droit de Paris. Conférences 1932, Paris, Domat, 1932, p. 65-108). Sur Pierre Saintyves (en réalité le pseudonyme de Pierre Nourry) (1870-1935), éditeur et libraire parisien, président d'honneur de la Société de folklore français, directeur de la Revue de folklore français et de folklore colonial et de la Revue anthropologique et maître de conférences à l'École d'anthropologie de Paris, cf. N. Belmont, Paroles païennes : mythe et folklore, op. cit.

I6o É. Jobbé-Duval, Les morts malfaisants. Larves, lémures, d'après le droit et les croyances populaires des Romains, Paris, Sirey, 1924 [rééd. Chambéry, éd. Exergue, 2000], p. 17. Précisons que les trois premiers chapitres de l'ouvrage étaient d'abord parus sous forme d'articles dans la Revue historique de droit français et étranger en 1923.

I6I Ibidem, p. I5.

162 Charles Appleton, par exemple, écrit également, quelques années plus tôt, que « dans l'histoire de l'humanité, la croyance au monde invisible a joué un rôle dont la science commence enfin à comprendre l'importance sans égale » (De quelques problèmes relatifs à l'bistoire du mariage. I. L'bypothèse d'une promiscuité primitive, Paris, E. de Boccard, I9I6, p. 24). Ce texte est un tiré-à-part d'un article paru dans la Revue générale du droit en i9i6. 
des défunts consiste à démontrer l'existence d'un invariant dans les mentalités primitives relatives à la mort.

43. Cet ouvrage novateur connaît, à sa sortie, un large accueil, émanant tant des milieux juridiques qu'extra-juridiques. Toutes les critiques s'accordent à reconnaître la grande originalité de l'étude de Jobbé-Duval, qui venait de renouveler entièrement un vieux sujet grâce à la méthode de l'histoire comparée du droit ${ }^{163}$. Recensant le premier l'ouvrage, Marcel Déroulède relève l'importante contribution au folklore juridique qu'il constitue. Il analyse l'opus comme un approfondissement des travaux de Lucien Lévy-Bruhl, tendant à démontrer que la mentalité « prélogique » des primitifs mise en lumière par ce dernier trouve des prolongements dans les sociétés civilisées, et en particulier chez les Bretons contemporains, ce que Jobbé-Duval met en lumière en mobilisant le concept de survivance $^{164}$. Quant à Joseph Lefort, il discute longuement l'ouvrage en le qualifiant de « magistrale suite des travaux de l'École anthropologique anglaise » incarnée par James Frazer et des travaux de Lucien Lévy-Bruhl' ${ }^{65}$. Sa recension prend d'ailleurs la forme d'un article de fond, preuve de l'impor tance qu'il accorde à l'ouvrage : il la reproduit presque à l'identique dans la Revue anthropologique en $1926^{166}$. La Revue historique de droit français et étranger se montre cependant un peu plus circonspecte sur un ouvrage qui décontenance quelque peu les historiens du droit romain par rapport à la majorité de la production du temps, plus volontiers technicienne. Si la revue rend hommage à l'érudition étendue de l'auteur et aux vues aussi neuves qu'ingénieuses proposées, elle en déplore également les analyses contradictoires, les hypothèses hasardeuses et une argumentation parfois peu convaincante ${ }^{167}$. Des critiques identiques sont émises par la Revue archéologique, qui, relevant à son tour l'originalité de l'ouvrage, le juge cependant trop dépendant de « travaux modernes » émis par des auteurs n'ayant pas toujours eux-mêmes travaillé de première main ${ }^{168}$. C'est ici une critique du caractère philologique des travaux mobilisés par Jobbé-Duval qui est avancée, à l'heure où le travail de terrain commence à gagner droit de cité parmi les sciences sociales. Le folkloriste Pierre Saintyves, pour sa part, attire également l'attention sur la méthode intertextuelle de l'auteur, tout en pointant quelques lacunes bibliographiques du romaniste ${ }^{169}$.

44. La postérité de l'ouvrage dépasse largement le XIX siècle, pour se poursuivre longtemps après le décès de Jobbé-Duval. Dans la seconde moitié du XXe siècle, l'anthropologue du droit Michel Alliot l'analyse comme l'« un des premiers exemples d'interprétation ethnologique des anciennes pratiques magico-religieuses ${ }^{170} \gg$. C'était là, cependant, rabattre l'ouvrage de Jobbé-Duval sur l'utilisation de la méthode ethnologique en histoire du droit. Dans la réédition abrégée qu'il donne de l'ouvrage en 2000 , l'expert en littérature et civilisation germaniques médiévales Claude Lecouteux (spécialiste de la question de la revenance) propose une analyse plus extensive des Morts malfaisants. Affirmant que cet ouvrage précurseur «n'a pas pris une ride depuis sa parution en 1924 », il en fait «l'une des toutes premières analyses scientifiques des morts malfaisants ». Et Claude Lecouteux d'affirmer, relevant l'approche pluridisciplinaire de l'auteur, fondée sur une connaissance du droit, de l'histoire, de la philologie et de l'ethnologie, que « Jobbé-Duval est, à sa façon, un historien des

I63 R. Cagnat, « Livres nouveaux. Émile Jobbé-Duval, Les morts malfaisants (Larvae, Lémures) d'après le droit et les croyances populaires des Romains, 1924 », Journal des savants, 23, juillet-août 1925, p. 179-I80.

I64 M. Déroulède, «Bibliographie. Émile Jobbé-Duval, Les morts malfaisants d'après le droit et les croyances populaires des Romains (1924) », Revue critique de législation et de jurisprudence, 44, 1915-1924, p. 595-596.

I65 J. Lefort, « Les morts malfaisants et les traditions romaines », Revue générale du droit, 50, 1926, p. I8-2I (p. 2I pour la citation).

I66 Id., « Les morts malfaisants et les traditions romaines », Revue anthropologique, 36, 1926, p. 372-376.

I67 A. Fliniaux, « Comptes rendus critiques. I. Droit romain. E. Jobbé-Duval, Les morts malfaisants, 1924 », Revue historique de droit français et étranger, 5, 1926, p. II6-I2I.

I68 S. R., « Bibliographie. Émile Jobbé-Duval, Les morts malfaisants, larvae, lémures d'après le droit et les croyances populaires des Romains, 1924 », Revue archéologique, XX, juillet-octobre 1924, p. 28I.

I69 P. Saintyves, « Compte rendu d'Émile Jobbé-Duval, Les morts malfaisants. Larves, lémures, d'après le droit », art. cit.

I70 M. Alliot, « L'acculturation juridique », dans Ethnologie générale, op. cit., p. I240.

Clio@Thémis - nI5, 2019 
mentalités » et que «son étude relève sans nul doute de l'anthropologie culturelle ${ }^{171} \gg$. Étonnante postérité, et belle revanche sans doute, pour un romaniste oublié par ses pairs...

\section{Conclusion}

45. Le portrait intellectuel d'Émile Jobbé-Duval permet de relativiser l'affirmation de Norbert Rouland qui, se livrant à un bref historique de la naissance de l'anthropologie juridique, relevait en 1988 le silence français ${ }^{172}$. Non : les Anglo-saxons ne sont pas les seuls pionniers d'une réflexion anthropologique sur le droit. Même si la contribution des juristes français à la genèse de ce nouveau champ du savoir émergeant dans les années I860-I88o est moins visible que les œuvres de synthèse retentissantes qui furent celles des ténors de la décennie prodigieuse, elle n'est pas, pour autant, inexistante. Dès la seconde moitié du XIX siècle, les questionnements anthropologiques innervent profondément la science juridique française, bousculent ses certitudes, interrogent sa méthode comme ses présupposés. Invalidant l'hypothèse d'une retard français en la matière, le cas Jobbé-Duval ouvre en réalité une lucarne sur un monde qui demeure à reconstituer : celui de la rencontre féconde entre droit et anthropologie. Indubitablement, celle-ci appelle d'autres études de cas qui permettront, à terme, une reconstitution fine et minutieuse du traitement du primitivisme juridique - et de sa signification - par les juristes des $\mathrm{XIX}^{\mathrm{e}}$ et $\mathrm{XX}^{\mathrm{e}}$ siècles.

\section{Laetitia GUERLAIN}

Université de Bordeaux

Institut de recherche Montesquieu

Centre Alexandre-Koyré - Histoire des sciences et des techniques (UMR 8560, EHESS/CNRS/ $\mathrm{MHNH}$

I7I C. Lecouteux, «Préface à la nouvelle édition », dans É. Jobbé-Duval, Les morts malfaisants. Larves, lémures, d'après le droit, op. cit., p. 6.

172 N. Rouland, Antbropologie juridique, Paris, Presses universitaires de France, 1988, p. 40. 